\title{
Data-driven Techniques to Estimate Parameters in the Homogenized Energy Model for Shape Memory Alloys
}

\author{
John H. Crews, ${ }^{1, *}$ Ralph C. Smith, ${ }^{2}$ Kyle M. Pender, ${ }^{3}$ \\ Jennifer C. Hannen, ${ }^{3}$ Gregory D. Buckner ${ }^{3}$ \\ ${ }^{1}$ Department of Mathematics, North Carolina State University \\ Raleigh, NC 27695, USA \\ ${ }^{2}$ Department of Mathematics, Center for Research in Scientific Computation \\ North Carolina State University, Raleigh, NC 27695, USA \\ ${ }^{3}$ Department of Mechanical and Aerospace Engineering \\ North Carolina State University, Raleigh, NC 27695, USA
}

\begin{abstract}
The homogenized energy model (HEM) is a unified framework for modeling hysteresis in ferroelectric, ferromagnetic, and ferroelastic materials. The HEM framework combines energy analysis at the lattice level with stochastic homogenization techniques, based on the assumption that quantities such as interaction and coercive fields are manifestations of underlying densities, to construct macroscopic material models. In this paper, we focus on the homogenized energy model for shape memory alloys (SMA). Specifically, we develop techniques for estimating model parameters based on attributes of measured data. Both the local (mesoscopic) and macroscopic models are described, and the model parameters' relationship to the material's response are discussed. Using these relationships, techniques for estimating model parameters are presented. The techniques are applied to constant-temperature stress-strain and resistance-strain data. These estimates are used in two manners. In one method, the estimates are considered fixed and only the HEM density functions are optimized. For SMA, the HEM incorporates densities for the interaction and relative stress, the width of the hysteresis loop. In the second method, the estimates are included in the optimization algorithm. Both cases are compared to experimental data at various temperatures, and the optimized model parameters are compared to the initial estimates.
\end{abstract}

\section{Introduction}

Shape memory alloys (SMA) are novel materials that exhibit two useful characteristics. The super-elastic effect allows the material to undergo significant strains without experiencing plastic deformation. The shape memory effect makes the material capable of recovering large strains upon heating, allowing for its use as an actuator. Furthermore, SMA's bio-compatibility and high energy density make the material suitable for in-vivo actuation applications, potentially revolutionizing biomedical devices.

SMA have been successfully incorporated into a number of prototypes and commercial applications. The super-elastic effect has been utilized in orthodontic wires, eye-glass frames, stents, and annuloplasty bands [23]. Applications using the shape memory effect include robotic catheters [33,34], robotic hands [22], jet chevrons $[9,10,26]$, and smart inhalers [21]. The design and control of these prototypes are complicated by the material's nonlinear, hysteretic dependence on stress and temperature. This complex behavior arises from the presence of three crystalline structures (or phases) in uniaxial applications. Computationally efficient, accurate models of the material's behavior are necessary for design optimization and control algorithms. Furthermore, the model adoption is greatly facilitated by easily identifying parameters, reducing the time required to achieve accurate model fits.

The homogenized energy model (HEM) is a unified framework for modeling SMA, ferroelectric, and ferromagnetic materials. The energy origin of the model was originally investigated for SMA by Müller and Wilmanksi [17] with further work by Achenbach, Seelecke [24, 25], and Huo [14]. The original models determined the equilibrium phase using the Gibbs energy to predict the mesoscopic (or single-crystal) behavior. The HEM framework for SMA builds on these models by homogenizing (or averaging) the local

\footnotetext{
*Author to whom correspondence should be addressed.

E-mail:jhcrews@ncsu.edu
} 
response to predict the macroscopic behavior of polycrystalline materials. The homogenization is accomplished by assuming that certain parameters are manifestations of underlying densities. In this manner, the model quantifies the inherent polycrystalline nature of SMA and other smart materials and is suitable for applications requiring the macroscopic actuator response.

The application of the HEM framework to SMA was originally reported in [15], and the model has also been applied to ferroelectric $[13,29,30,32]$ and ferromagnetic $[19,27,28]$ materials. A complete description of the unified framework is presented in $[26,31]$. Additionally, the HEM has been incorporated into a variety of control applications $[19,20]$. A computationally efficient implementation of the model is described in $[2,3]$.

In this paper, we focus on data-driven techniques for estimating model parameters. The relationship between the parameters and experimentally obtained stress, strain, and resistance data at various constant temperatures is discussed. These initial parameter estimates are used in two manners. In one method, the parameters are considered fixed while the density functions are optimized. In the second method, the parameters are incorporated into the optimization process with the density functions. Similar techniques for estimating parameters for ferroelectric and ferromagnetic models are presented in $[11,12]$.

The remainder of this paper is organized as follows. The SMA model is summarized in Section 2, including the local model based on the Gibbs energy and the homogenization techniques used to construct the macroscopic model. Techniques for identifying the model parameters from experimental data are detailed in Section 3. The two optimization problems are presented in Section 4. The experimental setup is described in Section 5, and experimentally obtained data is used to estimate the initial model parameters. Finally, the results of the optimized model are presented and compared to the experimental data in Section 6 .

\section{Homogenized Energy Model for SMA}

The complex behavior of SMA is due to the presence of three crystalline phases in uniaxial applications: austenite and two martensite phases. Austenite $(A)$ is induced by high temperatures, and the martensitic phases are induced by high stresses. Martensite plus $(M+)$ is stable under tensile stresses, and martensite minus $(M-)$ is stable under compressive stresses. The interaction of these phases yields the material's complex, thermo-mechanical response. The transition diagram for SMA is shown in Figure 1. Numerous models have been proposed to quantify the behavior of SMA, including both physical and phenomenological models. An overview of these models is presented in [26].

\subsection{Mesoscopic Model}

The homogenized energy model uses the free energy model to derive the kernel for the local (mesoscopic) material behavior. The free energy model of SMA uses phase fractions $\left(x_{A}, x_{M+}\right.$, and $\left.x_{M-}\right)$ to quantify the

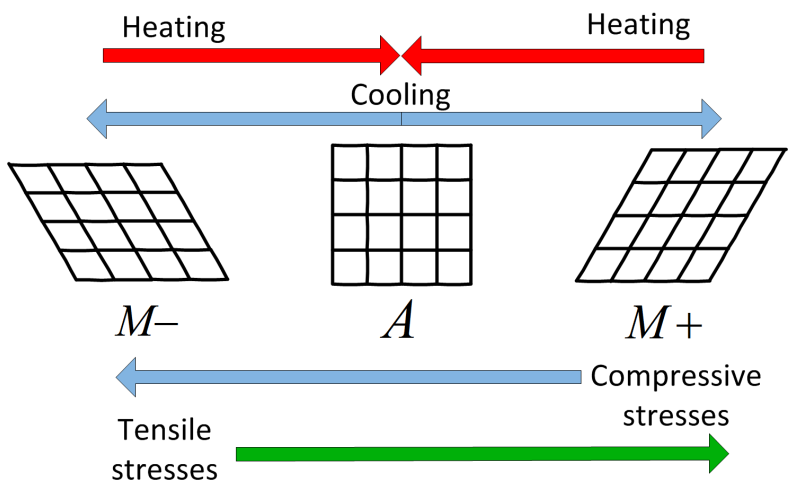

Figure 1: Uniaxial SMA phase transitions. 
proportion of the material in each of the three phases. The equilibrium phase fraction depends on the Gibbs energy

$$
G(\sigma, \varepsilon, T)=\psi(\varepsilon, T)-\sigma \varepsilon
$$

where the Helmholtz energy

$$
\psi(\varepsilon, T)= \begin{cases}\frac{1}{2} E_{M}\left(\varepsilon+\varepsilon_{T}\right)^{2} & \varepsilon<-\varepsilon_{M}(T) \\ A(T) \varepsilon^{2}+B(T) \varepsilon+C(T) & -\varepsilon_{M}(T) \leq \varepsilon<-\varepsilon_{A}(T) \\ \frac{1}{2} E_{A} \varepsilon^{2}+\Delta \beta(T) & |\varepsilon| \leq \varepsilon_{A}(T) \\ A(T) \varepsilon^{2}-B(T) \varepsilon+C(T) & \varepsilon_{A}(T)<\varepsilon \leq \varepsilon_{M}(T) \\ \frac{1}{2} E_{M}\left(\varepsilon-\varepsilon_{T}\right)^{2} & \varepsilon>\varepsilon_{M}(T)\end{cases}
$$

is represented using piecewise quadratic polynomials. The five polynomials correspond to the three phases and the barriers between them. In (2), $E_{A}$ and $E_{M}$ are the elastic moduli of austenite and martensite, respectively, and $\varepsilon_{T}$ is the maximum recoverable strain. The temperature-dependent inflection points are given by

$$
\varepsilon_{A}(T)=\frac{\sigma_{A}(T)}{E_{A}}
$$

and

$$
\varepsilon_{M}(T)=\frac{\sigma_{M}(T)}{E_{M}}+\varepsilon_{T}
$$

and represent the regions where each of phase is stable, as shown in Figure 2.

The critical transition stresses are given by

$$
\sigma_{A}(T)=\sigma_{L}+\Delta \sigma_{T}\left(T-T_{L}\right)
$$

and

$$
\sigma_{M}(T)=\sigma_{A}(T)-\sigma_{R}
$$

where $\sigma_{L}$ is the transition stress from $A$ to $M+$ at temperature $T_{L}, \Delta \sigma_{T}$ is the hysteresis loop's temperaturedependence, and $\sigma_{R}$ is the relative stress difference in loading and unloading. The parameters listed in Table 1 can be related to experimental data.

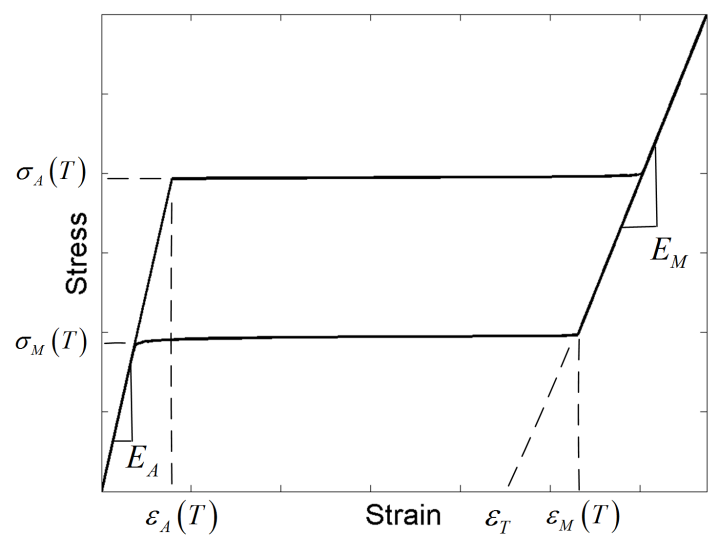

Figure 2: Relationship between the inflection points for austenite and martensite and the critical transition stresses. 
Table 1: SMA model parameters in the Gibbs energy and likelihood relations.

\begin{tabular}{cl}
\hline \hline Variable & Description \\
\hline$E_{A}$ & Austenite elastic modulus \\
$E_{M}$ & Martensite elastic modulus \\
$\sigma_{L}$ & Martensite transition stress at $T_{L}$ \\
$T_{L}$ & Lower transition temperature \\
$\sigma_{R}$ & Stress hysteresis loop width \\
$\Delta \sigma_{T}$ & Hysteresis loop's temperature dependence \\
$\varepsilon_{T}$ & Maximum recoverable strain \\
$\tau$ & Relaxation time \\
$V$ & Layer volume \\
\hline
\end{tabular}

The unknown coefficients in (2) are found by ensuring $C^{1}$ continuity, yielding

$$
\begin{gathered}
A(T)=\frac{E_{M}\left(\varepsilon_{T}-\varepsilon_{M}(T)\right)+E_{A} \varepsilon_{A}(T)}{2\left(\varepsilon_{A}(T)-\varepsilon_{M}(T)\right)}, \\
B(T)=\frac{\varepsilon_{A}(T)\left(E_{A}\left(\varepsilon_{T}-\varepsilon_{M}(T)\right)+E_{A} \varepsilon_{A}(T)\right)}{\varepsilon_{A}(T)-\varepsilon_{M}(T)}, \\
C(T)=\frac{2 \Delta \beta(T)\left(\varepsilon_{A}(T)-\varepsilon_{M}(T)\right)+\varepsilon_{A}(T)^{2}\left(E_{M}\left(\varepsilon_{T}-\varepsilon_{M}(T)\right)+E_{A} \varepsilon_{M}(T)\right)}{2\left(\varepsilon_{A}(T)-e \varepsilon_{M}(T)\right)},
\end{gathered}
$$

and

$$
\Delta \beta(T)=\frac{1}{2}\left(E_{M}\left(\varepsilon_{T}-\varepsilon_{A}(T)\right)\left(\varepsilon_{T}-\varepsilon_{M}(T)\right)-E_{A} \varepsilon_{A}(T) \varepsilon_{M}(T)\right) .
$$

The evolution of the phase fractions is governed by the coupled differential equations

$$
\begin{aligned}
& \dot{x}_{M+}(t)=-p_{+A} x_{M+}(t)+p_{A+} x_{A}(t) \\
& \dot{x}_{M-}(t)=-p_{-A} x_{M-}(t)+p_{A-} x_{A}(t) .
\end{aligned}
$$

The final phase fraction is found from the conservation equation

$$
1=x_{A}(t)+x_{M+}(t)+x_{M-}(t) .
$$

In (4), $p_{\alpha \beta}$ is the likelihood of transition from phase $\alpha$ to $\beta(\alpha, \beta=M+, M-$, and $A)$. The transition rates depend on the Gibbs energy and can be found using a number of methods. The original method developed by Achenbach [1] and Müller and Seelecke [16] is based on statistical mechanics and the sampling of the energy barriers by the equilibrium phases. Here, we assume that the transition rates depend on the activation energy, or the barriers in the energy landscape. Using the activation energy $\Delta G_{\alpha \beta}$, the transition rate is

$$
p_{\alpha \beta}(\sigma, T)=\frac{1}{\tau} \exp \left(-\frac{V \Delta G_{\alpha \beta}(\sigma, T)}{k T}\right)
$$

where $k$ is Boltzmann's constant.

The relaxation time $\tau$ and layer volume $V$ must be identified using experimental data and can be included in the optimization of model parameters. A schematic of the four barriers is shown in Figure 3 for a small applied stress and low temperature. The barriers $\Delta G_{\alpha \beta}(\sigma, T)$ are determined by finding the difference in minima and maxima of the polynomials in (1). For example,

$$
\Delta G_{-A}(\sigma, T)=G_{2}\left(\sigma, \varepsilon_{2}^{*}, T\right)-G_{1}\left(\sigma, \varepsilon_{1}^{*}, T\right),
$$




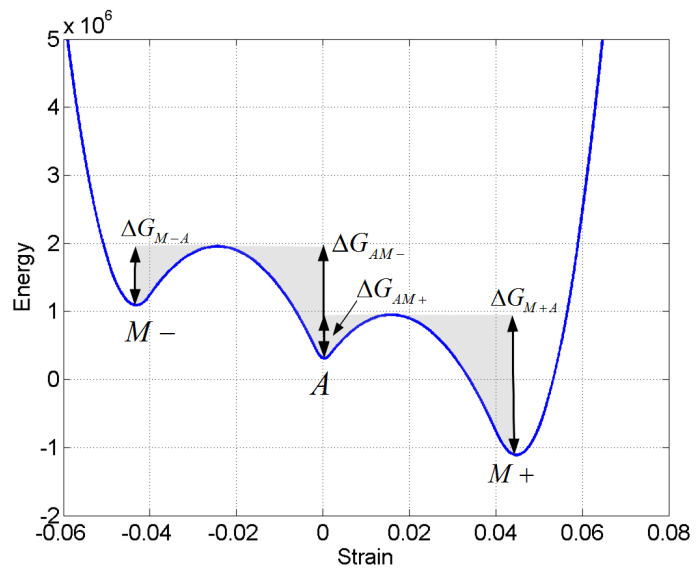

Figure 3: Gibbs energy landscape with barriers highlighted.

where $\varepsilon_{2}^{*}$ and $\varepsilon_{1}^{*}$ respectively solve

$$
\frac{\partial G_{2}(\sigma, \varepsilon, T)}{\partial \varepsilon}=0, \quad \frac{\partial G_{1}(\sigma, \varepsilon, T)}{\partial \varepsilon}=0 .
$$

The strain for a lattice element,

$$
\bar{\varepsilon}(t)=x_{A}(t)\left\langle\varepsilon_{A}\right\rangle+x_{M+}(t)\left\langle\varepsilon_{M+}\right\rangle+x_{M-}(t)\left\langle\varepsilon_{M-}\right\rangle,
$$

depends on the phase fractions and their respective equilibrium strains. Assuming low thermal activation, the equilibrium strains are given by

$$
\left\langle\varepsilon_{A}\right\rangle=\frac{\sigma(t)}{E_{A}}, \quad\left\langle\varepsilon_{M+}\right\rangle=\frac{\sigma(t)}{E_{M}}+\varepsilon_{T}, \quad\left\langle\varepsilon_{M-}\right\rangle=\frac{\sigma(t)}{E_{M}}-\varepsilon_{T} .
$$

The electrical resistance in SMA also depends on the phase fractions. In some applications, for example a wire, the resistance is useful for self-sensing, where the measured resistance is used to predict the strain in the wire [6-8]. As detailed in [6], the resistivity

$$
\rho_{s m a}(T)=x_{A}(t) \rho_{A}(T)+\left[x_{M+}(t)+x_{M-}(T)\right] \rho_{M}(T)
$$

depends on temperature, where the resistivity of each phase

$$
\begin{gathered}
\rho_{A}(T)=\rho_{0 A}\left(1+\alpha_{A} \Delta T\right) \\
\rho_{M}(T)=\rho_{0 M}\left(1+\alpha_{M} \Delta T\right)
\end{gathered}
$$

includes a nominal resistivity $\rho_{0 \beta}$ and the thermal dependence $\alpha_{\beta}$. The total resistance in the wire,

$$
\Omega(T, \varepsilon)=\frac{\rho_{s m a}(T) L(\varepsilon)}{A\left(\varepsilon_{e}\right)},
$$

accounts for the change in length of the wire due to strain

$$
L(\varepsilon)=L_{0}(1+\varepsilon(t))
$$


Additionally, the cross-sectional area

$$
A\left(\varepsilon_{e}\right)=\pi\left(R_{0}\left(1-\nu \varepsilon_{e}\right)\right)^{2}
$$

is corrected to account for elastic deformation, where the elastic strain is

$$
\varepsilon_{e}=\sigma(t)\left(\frac{x_{M+}(t)-x_{M-}(t)}{E_{M}}+\frac{x_{A}(t)}{E_{A}}\right) .
$$

Here, $L_{0}$ is the austenitic (zero strain) length of the wire, $R_{0}$ is the nominal wire radius, and $\nu$ is Poisson's ratio. Similar to $\tau$ and $V$, the resistance model parameters $\left(\rho_{0 M}, \rho_{0 A}, \alpha_{M}, \alpha_{A}\right.$ and $\left.\nu\right)$ must be estimated from experimental data and can be included in the optimization scheme as well.

In addition to the phase fraction ODEs (4) and the constitutive relations (6) and (9), an internal energy balance is necessary. The thermal evolution equation at the local level is

$$
\rho c_{v} \dot{T}(t)=-h\left(T(t)-T_{\infty}\right)-\kappa\left(T(t)-T_{e}(t)\right)+j(t)+\rho H\left(\dot{x}_{M+}(t)+\dot{x}_{M-}(t)\right),
$$

where $\rho$ is the SMA density, $c_{v}$ is the specific heat, $h$ is the convective heat transfer coefficient with ambient air temperature $T_{\infty}, \kappa$ is the conduction coefficient with surrounding temperature $T_{e}, j(t)$ is the electrical input power, and $H$ is the latent heat associated with the phase transformations. Here, we are using experimental data collected in a constant-temperature water bath and assume that the temperature change due to the latent heats is negligible due to the presence of a large thermal mass (water); hence, the thermal evolution equation is unnecessary.

The ODEs for the phase fractions (4) can be discretized and solved using an implicit Euler scheme. In the discretized form, the phase fractions $\vec{x}^{k+1}=\left[x_{M+}^{k+1}, x_{M-}^{k+1}\right]^{T}$, at time step $k+1$, are given by

$$
\vec{x}^{k+1}=\vec{x}^{k}+\Delta t\left[A\left(t^{k+1}\right) \vec{x}^{k+1}+b\left(t^{k+1}\right)\right],
$$

where

$$
A\left(t^{k+1}\right)=\left[\begin{array}{cc}
-p_{+A}\left(\sigma^{k+1}, T^{k+1}\right)-p_{A+}\left(\sigma^{k+1}, T^{k+1}\right) & -p_{A+}\left(\sigma^{k+1}, T^{k+1}\right) \\
-p_{A-}\left(\sigma^{k+1}, T^{k+1}\right) & -p_{-A}\left(\sigma^{k+1}, T^{k+1}\right)-p_{A-}\left(\sigma^{k+1}, T^{k+1}\right)
\end{array}\right]
$$

and

$$
b\left(t^{k+1}\right)=\left[\begin{array}{l}
p_{A+}\left(\sigma^{k+1}, T^{k+1}\right) \\
p_{A-}\left(\sigma^{k+1}, T^{k+1}\right)
\end{array}\right]
$$

The initial condition for the phase fractions depends on the temperature and stress; however, initializing experiments and simulations under zero stress simplifies the initial condition. At temperatures above the austenitic start temperature of SMA, $T_{A_{s}}$, the initial phase fraction is assumed to be fully austenitic and $x_{M+}(0)=x_{M-}(0)=0$. The austenitic start temperature is the approximate temperature at which SMA would transition to austenite under zero stress and is available from manufacturer data. The value can also be determined experimentally by straining a wire and observing the temperature at which it begins to contract under zero stress. At lower temperatures, experimental results have shown a propensity to exist in the $M+$ phase, leading to a non-zero initial strain even after heating and cooling under zero stress [5]. This effect may be due to material inhomogeneities or the two-way shape memory effect. To quantify the non-zero initial strain, the initial condition is based on this strain value. At low temperatures, the initial strain is given by

$$
x_{M+}(0) \varepsilon_{T}-x_{M-}(0) \varepsilon_{T}=\varepsilon(0) .
$$

Substituting in the conservation relation (5) and setting the $x_{A}$ fraction to zero yields

$$
x_{M+}(0)=\frac{\varepsilon(0)+\varepsilon_{T}}{2 \varepsilon_{T}}, \quad x_{M-}(0)=1-x_{M+}(0) .
$$




\subsection{Homogenized Energy Model}

The mesoscopic or lattice-level model quantifies the single crystal behavior of SMA by assuming uniform properties and disregarding material and stress inhomogeneities; however, SMA is inherently polycrystalline. The HEM quantifies the macroscopic behavior by assuming that the relative stress $\sigma_{R}$ and interaction stress $\sigma_{I}$ are manifestations of underlying densities $\nu_{R}\left(\sigma_{R}\right)$ and $\nu_{I}\left(\sigma_{I}\right)$. The macroscopic strain includes these effects and is given by

$$
\varepsilon(\sigma(t), T(t))=\int_{0}^{\infty} \int_{-\infty}^{\infty} \nu_{R}\left(\sigma_{R}\right) \nu_{I}\left(\sigma_{I}\right) \bar{\varepsilon}\left(\sigma(t)+\sigma_{I}, T(t), \sigma_{R}\right) d \sigma_{I} d \sigma_{R} .
$$

The relative stress density changes the hysteresis loop width (the difference in the loading and unloading stress, as shown in Figure 4(a)). However, all kernels switch from austenite to martensite at the same stress (point $A$ ). The relative stress also affects the height of the barrier between $M+$ or $M-$ and $A$ in the Gibbs energy landscape; see Figure 4(c).

The interaction stress density affects both loading (switching from austenite to martensite) and unloading (switching from martensite to austenite). For a given temperature and applied stress, the interaction stress density shifts the hysteresis loops up and down (Figure 4(b)), but all kernels have the same width and the same parameters. As illustrated in Figure 4(d), this behavior corresponds to a tilt in the Gibbs energy since the interaction stress changes the effective stress.

Both densities are necessary to accurately model the major loop and minor loop behavior. Without the interaction density, all kernels switch from austenite to martensite at the same stress (between points $A$ and $B$ in Figure $5(\mathrm{a})$ ). The only changes occur on unloading as the material switches from martensite to austenite (between points $C$ and $D$ in Figure 5(a)) over a range of stresses corresponding to the different relative stresses.

Without the relative stress density, the minor loops will be anhysteretic, as illustrated in Figure 5(b). The kernels begin switching from austenite to martensite at point $A$. If the stress is decreased from point $B$ to $C$, all of the kernels that switched to martensite will remain in martensite. Since all kernels have the same relative stress $\sigma_{R}$, the stress will have to decrease to approximately point $D$ before switching back to austenite will occur. Therefore, a minor loop between points $B$ and $C$ will be anhysteretic (hysteresis-free), which is contrary to the observed behavior.

The relative stress and interaction stress densities can take multiple forms, subject to certain conditions. Both densities must be integrable. The relative stress density is defined only for $\sigma_{R}>0$, since the switch from austenite to martensite occurs at a higher stress than the reverse transition. Furthermore, the interaction stress density is assumed to be symmetric. In [4], we compare two methods for representing the densities. One method uses a log-normal probability density function for $\nu_{R}\left(\sigma_{R}\right)$ and a normal density for $\nu_{I}\left(\sigma_{I}\right)$. The other method uses a linear combination of underlying log-normal and normal probability density functions. Here, we use the second method as it provides greater flexibility and accuracy. The two densities are then expressed as

$$
\nu_{R}\left(\sigma_{R}\right)=\frac{1}{C_{1}} \sum_{m=1}^{M_{\alpha}} \sum_{k=1}^{K_{\alpha}} \alpha_{k, m} \phi_{k, m}\left(\sigma_{R}\right) \quad C_{1}=\sum_{m=1}^{M_{\alpha}} \sum_{k=1}^{K_{\alpha}} \alpha_{k, m}
$$

and

$$
\nu_{I}\left(\sigma_{I}\right)=\frac{1}{C_{2}} \sum_{k=1}^{K_{\beta}} \beta_{k} \psi_{k}\left(\sigma_{I}\right) \quad C_{2}=\sum_{k=1}^{K_{\beta}} \beta_{k},
$$

where the coefficients $\alpha_{k, m}$ and $\beta_{k}$ are identified by optimizing the model response to observed experimental data. The coefficients $C_{1}$ and $C_{2}$ ensure that the densities integrate to unity. The basis functions $\phi_{k, m}\left(\sigma_{R}\right)$ and $\psi_{k}\left(\sigma_{I}\right)$ are given by

$$
\phi_{k, m}\left(\sigma_{R}\right)=\frac{1}{c_{k} \sigma_{R} \sqrt{2 \pi}} \exp \left(-\left[\ln \left(\sigma_{R}\right)-\mu_{R_{m}}\right]^{2} / 2 c_{k}^{2}\right)
$$


and

$$
\psi_{k}\left(\sigma_{I}\right)=\frac{1}{b_{k} \sqrt{2 \pi}} \exp \left(-\sigma_{I}^{2} / 2 b_{k}^{2}\right) .
$$

Initial estimates for the relative stress log-normal mean $\mu_{R}$ and standard deviation $c$ and interaction stress standard deviation $b$ are obtained from experimental data. The initial estimates are then perturbed to obtain the desired basis function parameters $\mu_{R_{m}}, c_{k}$ and $b_{k}$. For example, after estimating the standard deviation for the interaction stress, $b$, four additional basis function are created to have standard deviations of $0.25 b, 0.5 b, 2 b$, and $4 b$. The parameters $\mu_{R_{m}}$ and $c_{k}$ are perturbed in a similar manner about experimentallydetermined value of $\mu_{R}$ and $c$.

The macroscopic model (10) is integrated using 4-point Gaussian quadrature on 20 equal intervals. The discretized model is evaluated using

$$
\varepsilon(\sigma(t), T(t))=\sum_{i=1}^{N_{i}} \sum_{j=1}^{N_{j}} \nu_{R}\left(\sigma_{R_{i}}\right) \nu_{I}\left(\sigma_{I_{j}}\right) \bar{\varepsilon}\left(\sigma(t)+\sigma_{I_{j}}, T(t), \sigma_{R_{i}}\right) w_{i} w_{j},
$$

where the weights $w_{i}$ and $w_{j}$ are determined by Gaussian quadrature. The summations in (15) are easily

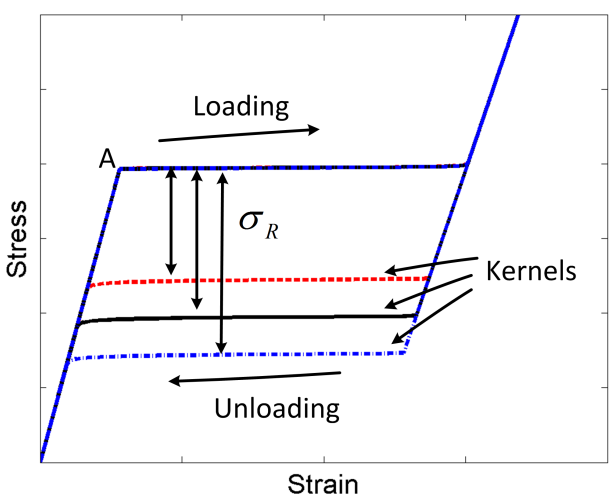

(a)

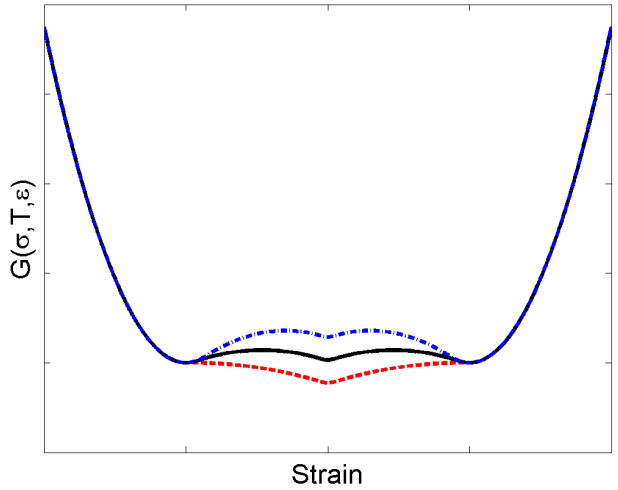

(c)

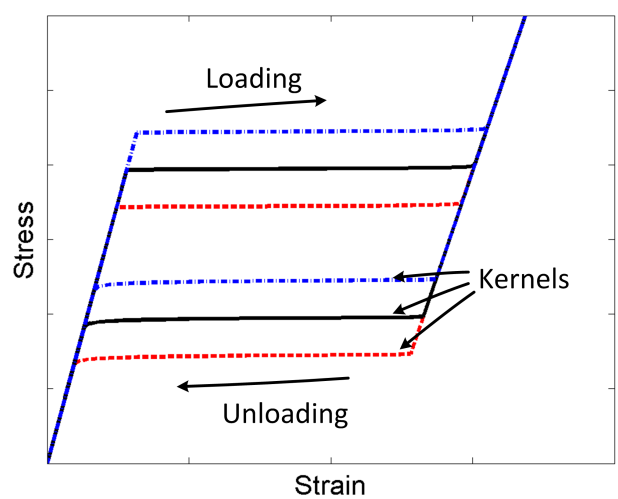

(b)

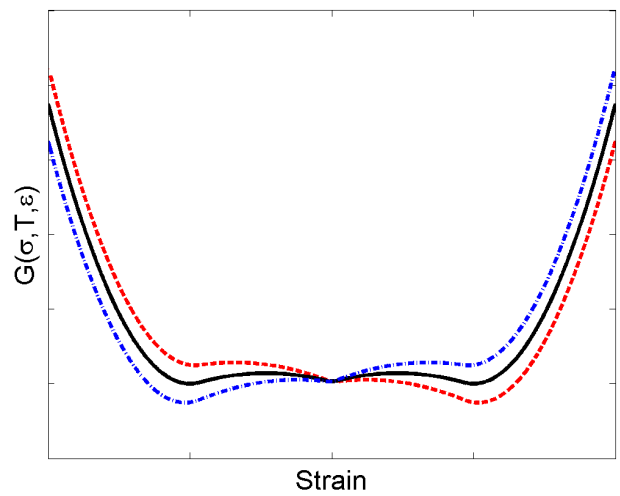

(d)

Figure 4: Homogenenized energy model kernels for different values of (a) $\sigma_{R}$ and (b) $\sigma_{I}$. Gibb's energy landscape for different values of (c) $\sigma_{R}$ and (d) $\sigma_{I}$. 


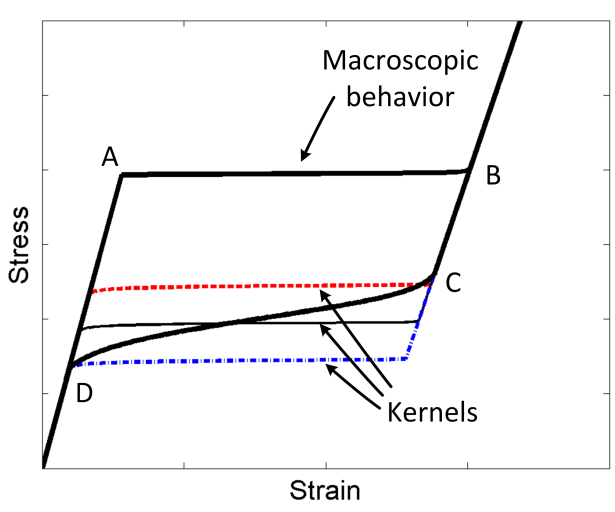

(a)

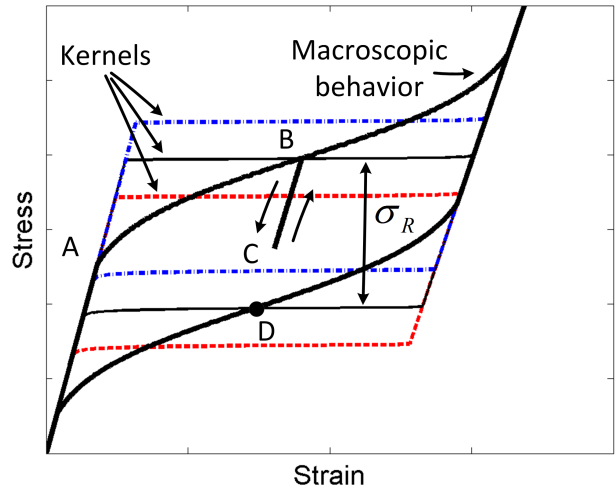

(b)

Figure 5: Hysteresis behavior when (a) $\nu_{R}\left(\sigma_{R}\right)$ is distributed but $\nu_{I}\left(\sigma_{I}\right)$ is constant, and (b) $\nu_{I}\left(\sigma_{I}\right)$ is distributed but $\nu_{R}\left(\sigma_{R}\right)$ is constant.

represented as matrix multiplications, increasing the computational efficiency of the model. In matrix form, the discrete model is

$$
\varepsilon(\sigma(t), T(t))=V^{T} \Gamma W
$$

where

$$
V^{T}=\frac{1}{d_{1}}\left[w_{1} \nu_{R}\left(\sigma_{R_{1}}\right), \cdots, w_{N_{i}} \nu_{R}\left(\sigma_{R_{N_{i}}}\right)\right]
$$

and

$$
W^{T}=\frac{1}{d_{2}}\left[w_{1} \nu_{I}\left(\sigma_{I_{1}}\right), \cdots, w_{N_{j}} \nu_{I}\left(\sigma_{I_{N_{j}}}\right)\right]
$$

incorporate the quadrature weights and densities evaluated at the quadrature points. The matrix

$$
[\Gamma]_{i j}=\bar{\varepsilon}\left(\sigma(t)+\sigma_{I_{j}}, T(t), \sigma_{R_{i}}\right)
$$

is the strain kernel evaluated at the quadrature points.

\section{Data-driven Techniques to Determine Initial Parameter Esti- mates}

\subsection{Mesoscopic Model Parameters}

One of the advantages of the homogenized energy model is that the model parameters listed in Table 1 can be related directly to experimental data. Stress, strain, resistance, and temperature data can be used to estimate these parameters. These estimates can either be used directly as the model parameters or as initial estimates for optimization algorithms. Accurate initial parameter estimates greatly enhance optimization convergence. Furthermore, accurate initial conditions may help overcome the likelihood of converging to local minima instead of the global minimum.

Many of the parameters can be directly estimated from stress-strain or resistance-strain data. For example, the elastic moduli $E_{A}$ and $E_{M}$ are determined from high temperature (above the austenite start temperature $T_{A_{S}}$ ) stress-strain data, as shown in Figure 6. The austenitic elastic modulus $E_{A}$ is the slope of the stress-strain curve as the SMA wire is initially loaded (line $\overline{a b}$ in Figure 6 ) . The martensitic elastic 


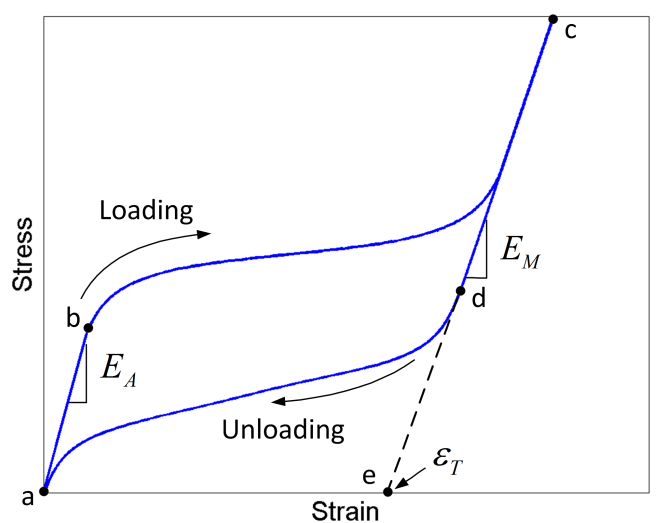

Figure 6: Relationship between the stress-strain response of SMA and model parameters $E_{A}$ (line $\overline{a b}$ ), $E_{M}$ (line $\overline{c d}$ ), and $\varepsilon_{T}$ (point $e$ ).

modulus is the slope of the stress-strain curve at high stresses during unloading (line $\overline{c d}$ in Figure 6 ). The maximum recoverable strain $\varepsilon_{T}$ is the intersection of $\overline{c d}$ with the zero stress axis (point $e$ in Figure 6).

The stress-strain hysteresis loop is temperature-dependent and satisfies the relation

$$
\sigma_{A}=\sigma_{L}+\Delta \sigma_{T}\left(T(t)-T_{L}\right)
$$

where $\sigma_{A}$ is the transition stress from austenite to martensite. The transition stress $\sigma_{L}$ and $T_{L}$ are coupled and not unique. Therefore, the values can be determined from the stress-strain response above $T_{A_{S}}$, as depicted in Figure 7. At a given temperature $T_{L}=T_{1}, \sigma_{L}$ is the stress at the midpoint of the transition from austenite to martensite (point $f$ in Figure 7). The transition stress from austenite to martensite varies linearly with temperature based on $\Delta \sigma_{T}$. An initial estimate for $\Delta \sigma_{T}$ is determined from the stress-strain response at two different temperatures. Given the stress at points $f$ and $g$ in Figure 7, it follows that

$$
\Delta \sigma_{T}=\frac{\sigma_{g}-\sigma_{f}}{T_{2}-T_{1}} .
$$

The relaxation time $\tau$ and volume layer $V$ are determined from creep data. Creep data is obtained by quickly loading the SMA to a specified strain $\hat{\varepsilon}_{1}$ and then holding the strain constant (point $a$ to point $b$ in Figure 8(a)). While the strain is held constant at $\hat{\varepsilon}_{1}$, the stress decreases (Figure 8(c)) as austenite converts to $M+$; see Figure $8(\mathrm{~d})$. Alternatively, the SMA can be loaded to a specified stress $\hat{(} \sigma)$ while the strain is monitored.

Assuming that during relaxation, the phase fraction transitions from $A$ to $M+$ only, the phase fraction ODE (4) reduces to

$$
\dot{x}_{M+}(t)=p_{A+}(\sigma, T) x_{A}(t)=p_{A+}(\sigma, T)\left(1-x_{M+}(t)\right) .
$$

Neglecting interaction and material inhomogeneity effects and assuming $t_{0}=0$ and $x_{M+}\left(t_{0}\right)=x_{M+_{0}}$, the analytical solution to (17) is

$$
x_{M+}(t)=\left(x_{M+o}+1\right) \exp \left(p_{A+}(\sigma, T) t\right)-1 .
$$

In (18), the transition rate $p_{A+}(\sigma, T)$ depends on the the stress; however, it is assumed that at strains near the maximum recoverable strain $\varepsilon_{T}$ the energy barrier $\Delta G_{A+}(\sigma, T)$ has been eliminated, yielding

$$
p_{A+}(\sigma, T)=\frac{1}{\tau} \exp \left(-\frac{V \Delta G_{A+}(\sigma, T)}{k_{b} T}\right)=\frac{1}{\tau} .
$$


The assumption that the energy barrier is zero eliminates the stress dependence and the volume layer $V$, leaving the unknown model parameter $\tau$. As shown in Figure 9, as the material begins switching from $A$ to $M+($ point $a)$, the energy barrier is large and its effect on the transition rate cannot be neglected. However, at strains near $\varepsilon_{T}$ (point $b$ ), the energy barrier is nearly eliminated. Furthermore, we note that

$$
\frac{V}{k_{b} T}<<1,
$$

providing further justification for the assumption

$$
\exp \left(-\frac{V \Delta G_{A+}(\sigma, T)}{k T}\right) \approx 1
$$

at strains near $\varepsilon_{T}$. Rate effects in this region are attributed solely to the relaxation time $\tau$.

Using the measured creep data, we assume that the decay in stress is proportional to the decay in austenite (or the increase in $M+$ since the ODEs are developed using $M+$ and $M-$ ). Letting $d_{1}$ and $d_{2}$ denote the change in stress shown in Figure 10(a) and using the assumption that the decay rate for stress is equal to the increase in $M+$, we obtain

$$
\frac{x_{M+}\left(t_{2}\right)-x_{M+}\left(t_{3}\right)}{x_{M+}\left(t_{1}\right)-x_{M+}\left(t_{2}\right)}=\frac{\sigma_{3}-\sigma_{2}}{\sigma_{2}-\sigma_{1}}=\frac{d_{2}}{d_{1}} .
$$

Assuming constant time steps $\Delta t$ and using (18), Equation (20) simplifies to

$$
\frac{\exp \left(-2 \cdot \Delta t \cdot p_{A+}(\sigma, T)\right)-\exp \left(-3 \cdot \Delta t \cdot p_{A+}(\sigma, T)\right)}{\exp \left(-\Delta t \cdot p_{A+}(\sigma, T)\right)-\exp \left(-2 \cdot \Delta t \cdot p_{A+}(\sigma, T)\right)}=\frac{d_{2}}{d_{1}}
$$

Solving for $p_{A+}(\sigma, T)$ yields

$$
p_{A+}(\sigma, T)=\frac{1}{\Delta t} \ln \left(\frac{d_{1}}{d_{2}}\right)
$$

Substituting the simplified transition likelihood (19) gives

$$
\frac{1}{\tau}=\frac{1}{\Delta t} \ln \left(\frac{d_{1}}{d_{2}}\right)
$$

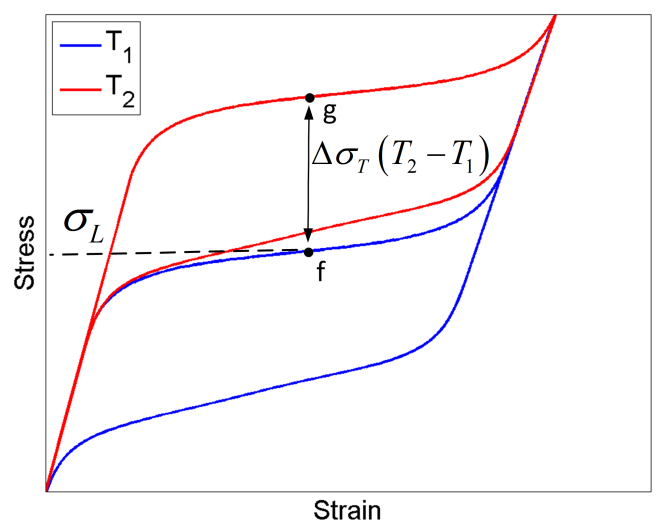

Figure 7: Relationship between the stress-strain response of SMA and model parameters $\sigma_{L}$ (point $f$ ), $T_{L}$ (temperature corresponding to stress at point $f$ ), and $\Delta \sigma_{T}$ (change in stress observed between $f$ and $g$ over change in temperature $T_{2}-T_{1}$ ). 


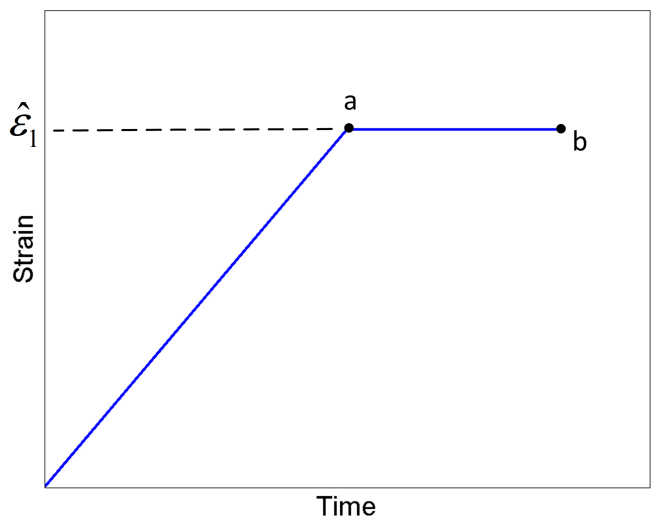

(a)

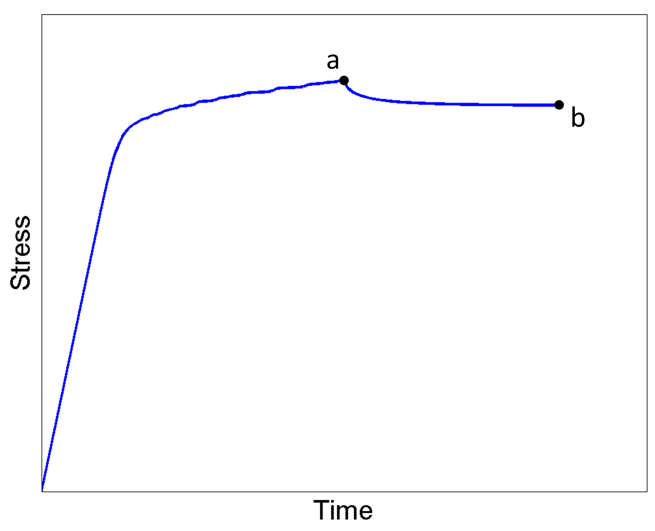

(c)

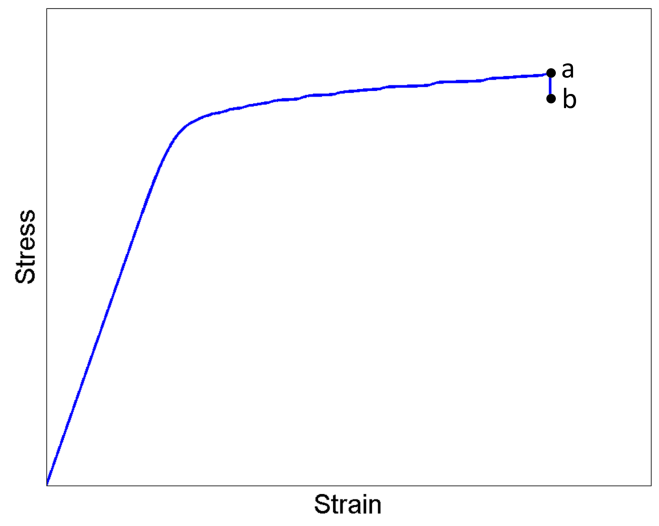

(b)

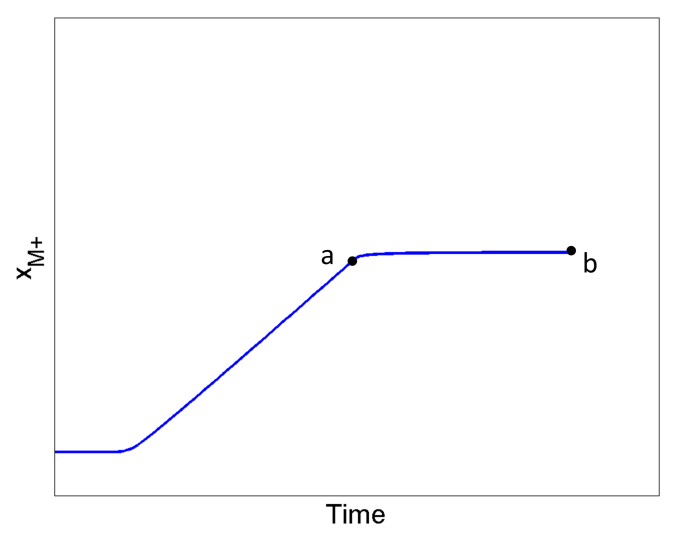

(d)

Figure 8: Creep data used to obtain estimates of $\tau$ and $V$ : (a) strain input (constant from $a$ to $b$ ); (b) resulting stress-strain response; (c) stress decay from $a$ to $b$ as strain is held constant; (d) assumed increase in $M+$ phase fraction as strain is held constant.

After solving for $\tau$, creep data at a lower strain (point $a$ in Figure 9(a)) is used to determine the volume layer $V$. The same process is used, except

$$
p_{A+}(\sigma, T)=\frac{1}{\tau} \exp \left(-\frac{V \Delta G_{A+}(\sigma, T)}{k_{b} T}\right)=\frac{1}{\Delta t} \ln \left(\frac{d_{1}}{d_{2}}\right)
$$

is solved for $V$. The barrier $\Delta G_{A+}(\sigma, T)$ depends on the stress; however, (23) is derived assuming the stress is constant over this interval, which is not the case. The stress at the midpoint can be used or Equation (21) can be solved for $V$ using the stresses at $t_{1}, t_{2}$, and $t_{3}$.

The resistance model parameters for martensite are estimated using the measured resistance at different low temperatures. At low temperatures, the equilibrium phase is $M+$ after loading and unloading. At this point (a strain of $\varepsilon_{T}$ and point $a$ in Figure 11(a)), effects of $\alpha_{M}$ and $\nu$ are negated. The resistivity is

$$
\rho_{M 0}=\frac{\Omega_{a} \pi R_{0}^{2}}{L\left(\varepsilon_{e_{a}}\right)},
$$


where $\Omega_{a}$ is the measured resistance and $R_{0}$ is the SMA radius. The Poisson's ratio is calculated using the low-temperature resistance at two different strains and by solving

$$
\Omega_{b}-\Omega_{b}=\rho_{M 0}\left(\frac{L\left(\varepsilon_{e_{b}}\right)}{\pi\left(R_{0}-R_{0} \nu \varepsilon_{b}\right)^{2}}-\frac{L\left(\varepsilon_{e_{a}}\right)}{\pi R_{0}^{2}}\right)
$$

for $\nu$. The austenite nominal resistivity is determined using high-temperature resistance strain data shown

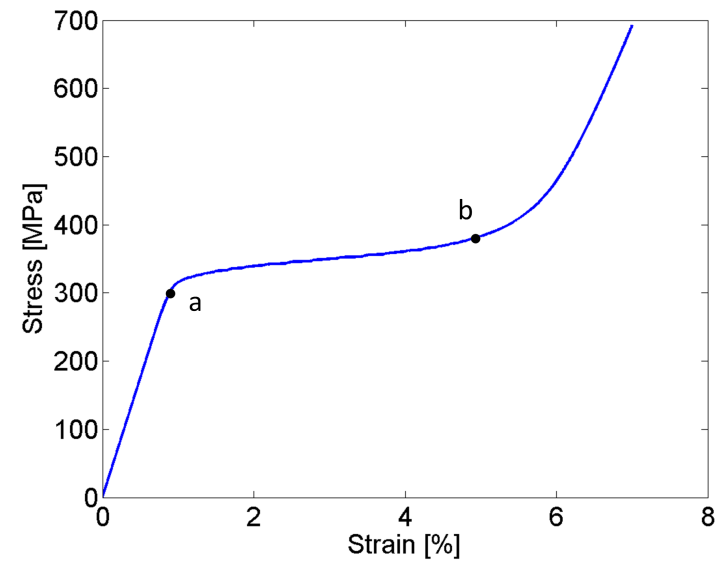

(a)

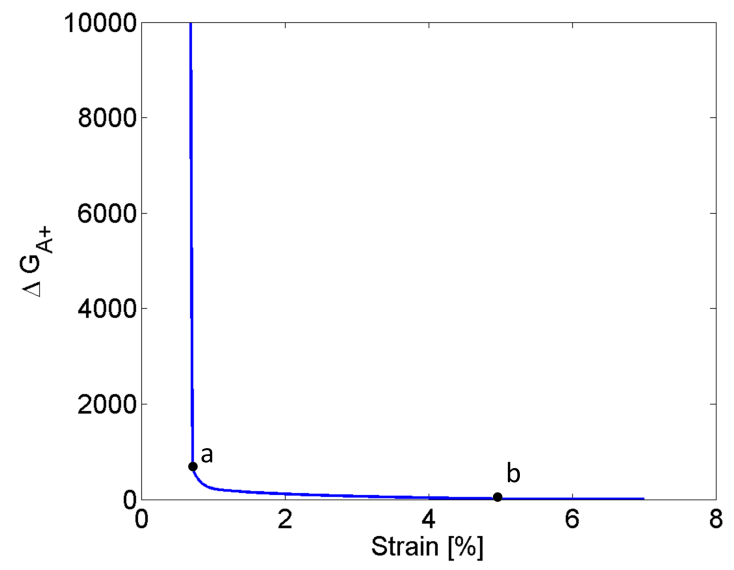

(b)

Figure 9: (a) High-temperature response of SMA during loading using $\tau=0.1 \mathrm{~s}$ and $V=5 \times 10^{-23} \mathrm{~m}^{3}$; (b) energy barrier between $A$ and $M+$ during loading.

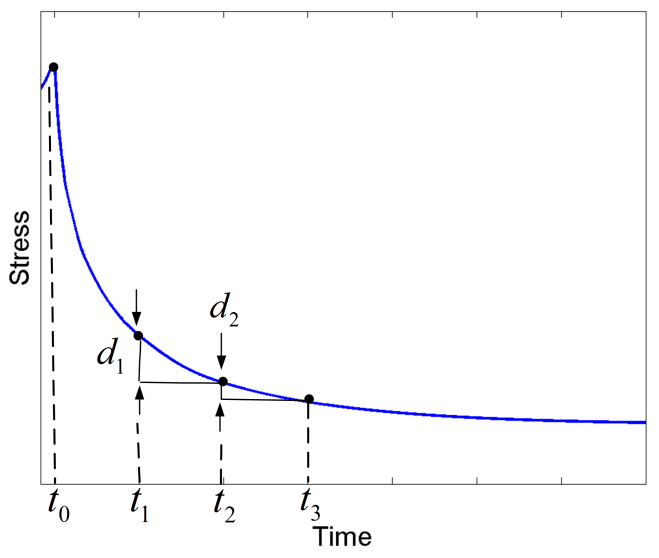

(a)

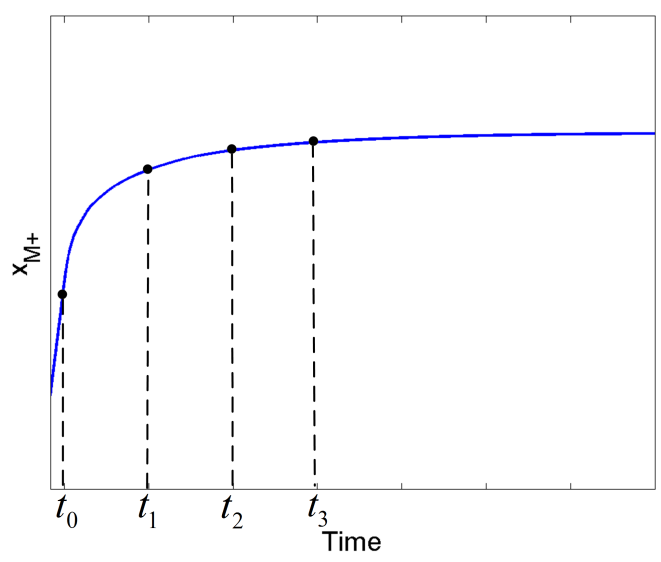

(b)

Figure 10: Estimation of $\tau$ : (a) stress decay during creep and change in stress $d_{1}$ and $d_{2}$ over equal time intervals; (b) corresponding increase in $M+$ phase fraction during creep. 


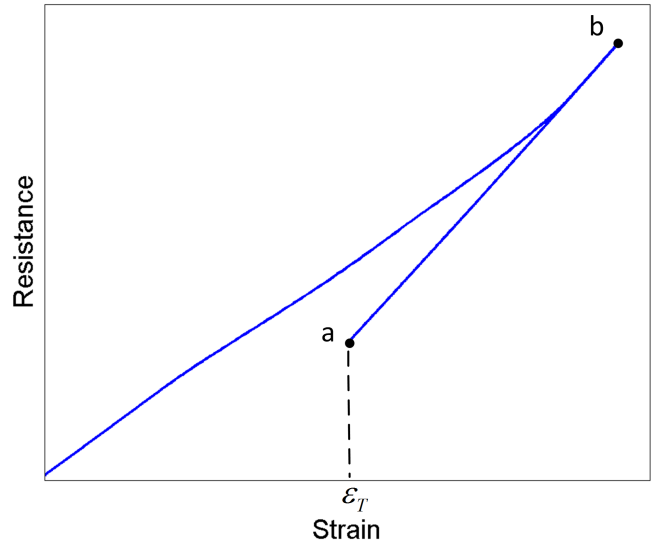

(a)

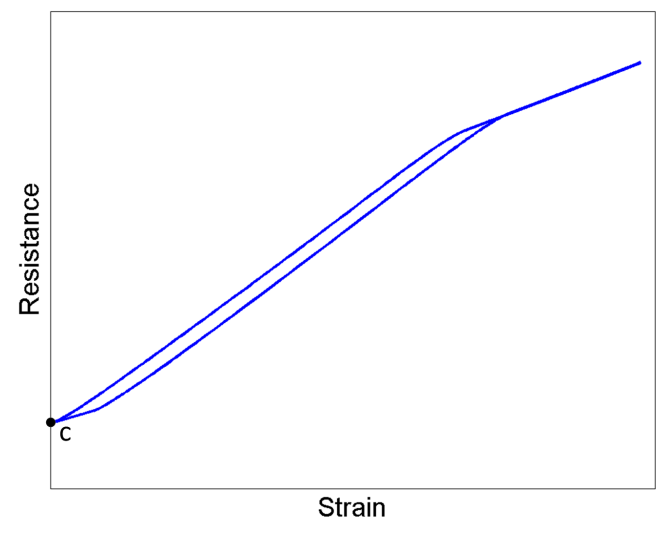

(b)

Figure 11: Estimation of resistance model parameters: (a) $\rho_{M 0}$ is estimated from the resistance at point $a$ and $\nu$ estimated using the change in resistance between points $b$ and $a$; (b) $\rho_{A 0}$ using high-temperature resistance-strain data (point $c$ ).

in Figure 11. Using the resistance at zero strain, the resistivity is

$$
\rho_{A 0}=\frac{\Omega_{c} \pi R_{0}^{2}}{L\left(\varepsilon_{e_{c}}\right)} .
$$

Note that this calculation assumes that the temperature dependence is based on a different nominal temperature than the martensite dependence.

As shown in Figure 12, the temperature dependence of the resistivities $\alpha_{M}$ and $\alpha_{A}$ can be found by varying the temperature under zero stress. After loading and unloading at low temperatures, the wire will be in $M+$. As the temperature rises, the resistance (and resistivity) rises due to $\alpha_{M}$. Near the austenite start temperature, the resistance drops, due to the lower resistivity of austenite. Further increases in temperature lead to increases in resistance due to $\alpha_{A}$. Using the points indicated in Figure 12(a), we obtain

$$
\alpha_{M}=\frac{\Omega_{2} A\left(\varepsilon_{e}\right) / L\left(\varepsilon_{e}\right)-\Omega_{1} A\left(\varepsilon_{e}\right) / L\left(\varepsilon_{e}\right)}{T_{2}-T_{1}}
$$

and

$$
\alpha_{A}=\frac{\Omega_{4} A\left(\varepsilon_{e}\right) / L\left(\varepsilon_{e}\right)-\Omega_{3} A\left(\varepsilon_{e}\right) / L\left(\varepsilon_{e}\right)}{T_{4}-T_{3}} .
$$

Alternatively, $\alpha_{M}$ and $\alpha_{A}$ can be identified using experimental resistance-strain data at two different temperatures after identifying $\rho_{M 0}, \rho_{A 0}$, and $\nu$. For example, the measured resistance at $d$ and $e$ in Figure 12 (b) can be used to find $\alpha_{A}$ using (7). Similarly, the measured resistance at $f$ and $g$ can be used to find $\alpha_{M}$ using (8).

\subsection{Homogenized Energy Model Parameters}

An initial estimate for the interaction stress standard deviation $b$ is obtained from high-temperature stressstrain data. As shown in Figure 5(b), the interaction stress causes the material to switch from $A$ to $M+$ over a range of stresses instead of at $\sigma_{A}$. Using this range of stress and assuming a normal distribution, approximately $95 \%$ of the kernels switch from $A$ to $M+$ between $\sigma_{A}-2 b$ and $\sigma_{A}+2 b$. Note that $\sigma_{A}$ is 
determined from (3) after estimating $\sigma_{L}, T_{L}$, and $\Delta \sigma_{T}$. Switching from $A$ to $M+$ begins where the stressstrain response deviates from elastic loading along $E_{A}$ and ends where the material elastically deforms along $E_{M}$; see Figure 13. After estimating the interaction stress standard deviation, the interaction stress density kernels (14) are initialized with multiples of $b$, yielding the desired number of $b_{k}$.

Using the switching region identified in Figure 13, the parameters for the relative stress density $\nu_{R}\left(\sigma_{R}\right)$ can be estimated using characteristics of log-normal densities [18]. The hysteresis loop width $\bar{\sigma}_{R}$ is estimated using the difference in loading and unloading at the midpoint of the major loop ( $\sigma_{A}$ on loading), as shown Figure 14. Assuming this value is the mode, the log-normal density mean is given by

$$
\mu_{R}=\ln \left(\bar{\sigma}_{R}\right) .
$$

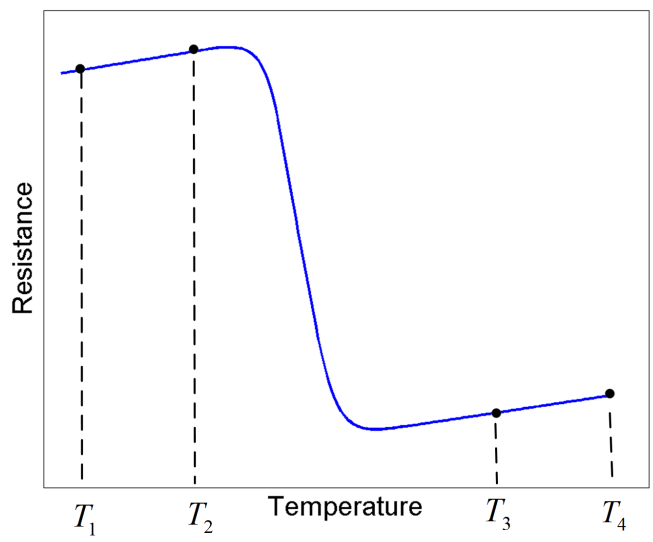

(a)

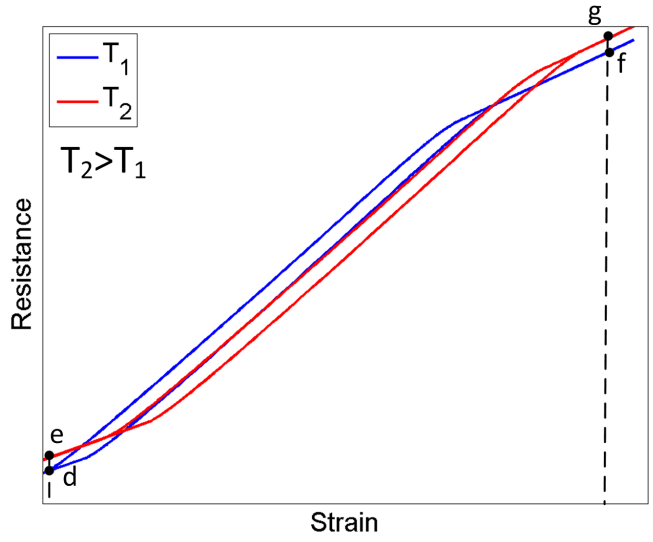

(b)

Figure 12: Estimation of $\alpha_{M}$ and $\alpha_{A}$ using resistance-temperature data: (a) resistance-temperature data at zero stress; (b) resistance-strain data at two different temperatures (points $d$ and $e$ for $\alpha_{A}$ and points $f$ and $g$ for $\left.\alpha_{M}\right)$.

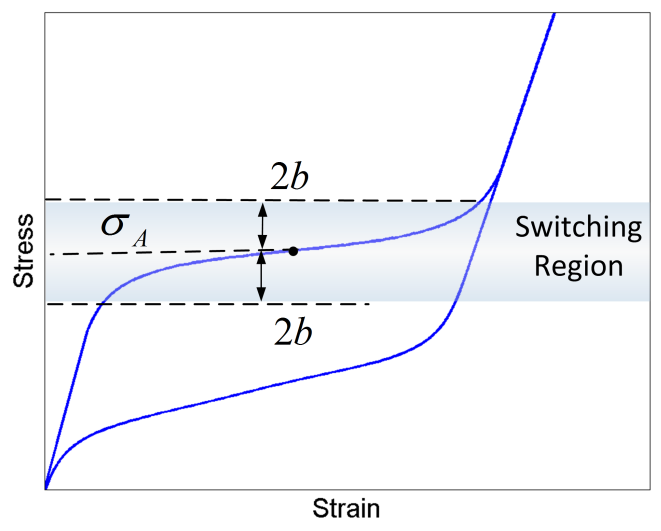

Figure 13: Relationship between the stress-strain response of SMA and the initial estimate for the interaction stress standard deviation $b$. 
On unloading, switching ends when $\nu_{I}\left(\sigma_{I}\right)$ stops intersecting with $\nu_{R}\left(\sigma_{R}\right)$ at the $95.4 \%$ confidence interval. Using this assumption and identifying the end of switching ( $\sigma_{F}$ in Figure 14), the standard deviation is

$$
c=\frac{1}{2}\left(\ln \left(\sigma_{F}-2 b\right)-\mu_{R}\right) .
$$

The relative stress density basis functions (13) are initialized with multiples of the macroscopic estimate for the log-normal mean $\mu_{R}$ and standard deviation $c$, which yields the desired values for $\mu_{R_{m}}$ and $c_{k}$.

An alternative method to identify the density parameters is to fit a log-normal and normal density directly to the experimental data. After identifying $\sigma_{A}$ and the switching region in Figure 13, the interaction stress standard deviation is

$$
b=\sqrt{\frac{1}{N_{k}-1} \sum_{k}^{N_{k}}\left(\hat{\sigma}^{k}-\sigma_{A}\right)^{2}},
$$

where $\hat{\sigma}^{k}$ is the experimentally measured stress within the switching region.

The experimentally observed relative stress

$$
\hat{\sigma}_{R}^{k}=\hat{\sigma}_{A}^{k}-\hat{\sigma}_{M}^{k}
$$

is estimated at each measured strain $\hat{\varepsilon}^{k}$ by taking the difference in the loading stress $\hat{\sigma}_{A}^{k}$ and unloading stress $\hat{\sigma}_{M}^{k}$. This method determines the experimentally observed relative stress throughout the entire major loop, as shown in Figure 15. Using this collection of estimates, the mean $\mathrm{E}\left(\hat{\sigma}_{R}^{k}\right)$ and variance $\operatorname{Var}\left(\hat{\sigma}_{R}^{k}\right)$ are calculated. Again using the characteristics of log-normal distributions, the density parameters are given by

$$
\mu_{R}=\ln \left(\mathrm{E}\left(\hat{\sigma}_{R}^{k}\right)\right)-\frac{1}{2} \ln \left(1+\frac{\operatorname{Var}\left(\hat{\sigma}_{R}^{k}\right)}{\mathrm{E}\left(\hat{\sigma}_{R}^{k}\right)^{2}}\right)
$$

and

$$
c^{2}=\ln \left(1+\frac{\operatorname{Var}\left(\hat{\sigma}_{R}^{k}\right)}{\mathrm{E}\left(\hat{\sigma}_{R}^{k}\right)^{2}}\right) .
$$

The methods for estimating the model parameters are summarized in Table 2. The methods described in this section and in Table 2 are not the only ways to identify the parameters. The parameters may be

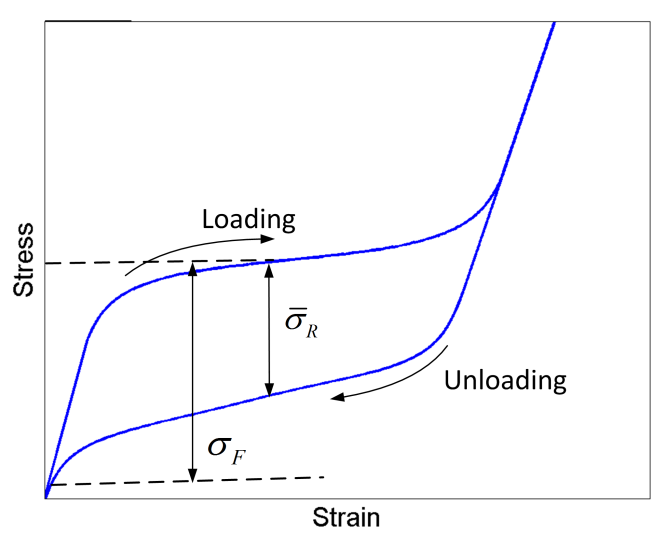

Figure 14: Relationship between the stress-strain response of SMA and the initial estimate for the relative stress $\hat{\sigma}_{R}$ and final switching stress $\sigma_{F}$. 


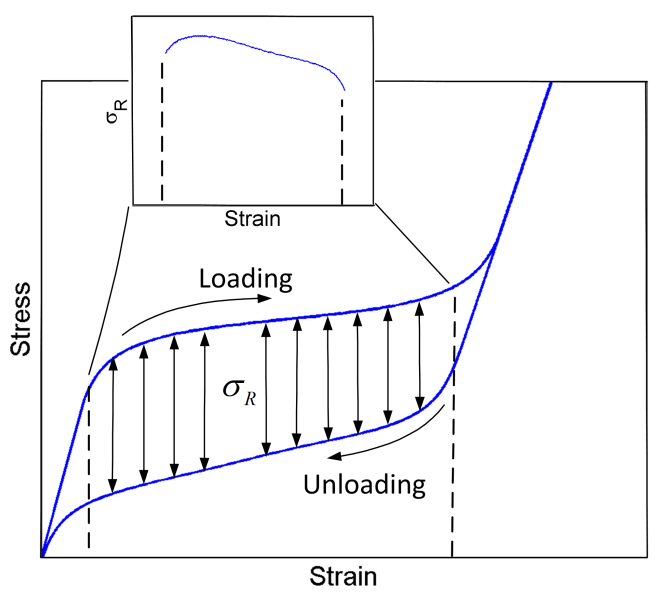

Figure 15: Relationship between the stress-strain response of SMA and the initial estimate for the relative stress $\sigma_{R}$.

estimated using a variety of tests; however, the summary presented in Table 2 attempts to minimize the experiments necessary to identify the parameters. Using these techniques requires five experiments, listed in Table 3. If a resistance model is unnecessary, then only four experiments are required.

\section{Parameter Optimization}

Two different optimization cases are presented and compared. In one case, only the densities $\nu_{R}\left(\sigma_{R}\right)$ and $\nu_{I}\left(\sigma_{I}\right)$ are optimized while the model parameters are considered constants identified from the experimental data. In the second case, the model parameters are considered initial estimates and included in the optimization algorithm with the densities. Both cases uses MATLAB's lsqnonlin algorithm.

\subsection{Densities Only}

Optimizing the density functions (11) and (12) involves finding the coefficients $\alpha_{k, m}$ and $\beta_{k}$ that minimize the sum of squared errors

$$
\begin{aligned}
F(\vec{q}) & =\frac{1}{2} \sum_{k=1}^{N_{k}} e\left(t^{k}\right)^{2} \\
& =\frac{1}{2} \sum_{k=1}^{N_{k}}\left(\hat{\varepsilon}^{k}-\varepsilon\left(\sigma\left(t^{k}\right), T\left(t^{k}\right)\right)\right)^{2} .
\end{aligned}
$$

between the measured strain $\hat{\varepsilon}^{k}$ and predicted strain $\varepsilon\left(\sigma\left(t^{k}\right), T\left(t^{k}\right)\right)$, where the design variables are

$$
\vec{q}=\left[\alpha_{1}, \cdots, \alpha_{K_{\alpha}, M_{\alpha}}, \beta_{1}, \cdots, \beta_{K_{\beta}}\right]^{T} .
$$

The analytical Jacobian is derived to decrease computational time for the optimization algorithm, as numerical approximations of the Jacobian require additional function evaluations and decreases accuracy. The 
Table 2: Data-driven techniques to determine initial parameter estimates.

\begin{tabular}{|c|c|c|}
\hline Parameter & Data & Source \\
\hline$E_{A}$ & Stress-strain data above $T_{A_{S}}$ & $\begin{array}{l}\text { Slope of the high-temperature stress-strain response } \\
\text { at small strain values; slope of line } \overline{a b} \text { in Fig. } 6 \text {. }\end{array}$ \\
\hline$E_{M}$ & Stress-strain data above $T_{A_{S}}$ & $\begin{array}{l}\text { Slope of the high-temperature stress-strain response } \\
\text { at high strain values; slope of line } \overline{c d} \text { in Fig. } 6 .\end{array}$ \\
\hline$\varepsilon_{T}$ & Stress-strain data above $T_{A_{S}}$ & $\begin{array}{l}\text { Intersection of the high-temperature martensite } \\
\text { load-line with the zero-stress axis; point } e \text { in Fig. } 6 .\end{array}$ \\
\hline$\sigma_{L}$ & Stress-strain data above $T_{A_{S}}$ & $\begin{array}{l}\text { Stress midpoint of a high-temperature major loop; } \\
\text { point } f \text { in Fig. } 7 \text {. }\end{array}$ \\
\hline$T_{L}$ & Stress-strain data above $T_{A_{S}}$ & Temperature at which $\sigma_{L}$ is estimated. \\
\hline$\Delta \sigma_{T}$ & $\begin{array}{l}\text { Stress-strain data at two differ- } \\
\text { ent temperatures above } T_{A_{S}}\end{array}$ & $\begin{array}{l}\text { Difference in the midpoint of the major loops at two } \\
\text { different temperatures; Eqn. (16). }\end{array}$ \\
\hline$\tau$ & $\begin{array}{l}\text { Creep data above } T_{A_{S}} \text { at a fixed } \\
\text { strain near } \varepsilon_{T}\end{array}$ & Eqn. $(22)$ \\
\hline$V$ & $\begin{array}{l}\text { Creep data above } T_{A_{S}} \text { at a fixed } \\
\text { strain near } 1 \%\end{array}$ & Eqn. (23). \\
\hline$b$ & Stress-strain data above $T_{A_{S}}$ & $\begin{array}{l}\text { Determine the } 95 \% \text { confidence interval over which } \\
\text { switching occurs (Fig. 13) and set equal to } \sigma_{A} \pm 2 b \\
\text { or fit an estimate for a normal density to the exper- } \\
\text { imental data (Eqn. }(31)) \text {. }\end{array}$ \\
\hline$\mu_{R}$ & Stress-strain data above $T_{A_{S}}$ & $\begin{array}{l}\text { Estimate the difference in loading and unloading at } \\
\text { the midpoint of the major loop (Eqn. (29)) or fit a } \\
\text { log-normal density to the experimental data using } \\
\text { the mean and variance (Eqn. }(32)) \text {. }\end{array}$ \\
\hline$c$ & Stress-strain data above $T_{A_{S}}$ & $\begin{array}{l}\text { Determined from the } 95 \% \text { confidence interval when } \\
\nu_{R}\left(\sigma_{R}\right) \text { and } \nu_{I}\left(\sigma_{I}\right) \text { stop interacting on unloading } \\
\text { (Eqn. }(30)) \text { or fit a log-normal density to the } \\
\text { experimental data using the mean and variance } \\
\text { (Eqn. }(33)) \text {. }\end{array}$ \\
\hline$\rho_{M 0}$ & $\begin{array}{l}\text { Resistance at a low temperature } \\
\text { and } \varepsilon_{T}\end{array}$ & Eqn. (24) \\
\hline$\rho_{A 0}$ & $\begin{array}{l}\text { Resistance at a high temperature } \\
\text { and zero strain }\end{array}$ & Eqn. (26) \\
\hline$\alpha_{M}$ & $\begin{array}{l}\text { Resistance-temperature data at } \\
\text { low temperatures and zero stress } \\
\text { or resistance-strain data at two } \\
\text { temperatures }\end{array}$ & Fig. 12(a) and Eqn. (27) or Fig. 12(b) \\
\hline$\alpha_{A}$ & $\begin{array}{l}\text { Resistance-temperature data at } \\
\text { low temperatures and zero stress } \\
\text { or resistance-strain data at two } \\
\text { temperatures }\end{array}$ & Fig. 12(a) and Eqn. (28) or Fig. 12(b) \\
\hline$\nu$ & $\begin{array}{l}\text { Resistance-strain data at low } \\
\text { temperature }\end{array}$ & Solve Eqn. (25) \\
\hline
\end{tabular}


Table 3: Ideal experiments for identifying model parameters.

\section{Description}

Stress-resistance-strain data at temperature $T_{1}>T_{A_{s}}$ with full major loop.

Stress-resistance-strain data at temperature $T_{2}>T_{1}$ with full major loop.

Creep data at temperature $T>T_{A_{s}}$ and $\varepsilon \approx \varepsilon_{T}$ : quickly load the wire to approximately $\varepsilon_{T}$ and then hold constant while recording stress.

Creep data at temperature $T>T_{A_{s}}$ and $\varepsilon \approx 1 \%$.

Resistance-strain data at temperature $T_{3}<T_{A_{s}}$ : heat the wire under zero stress and then cool; load to fully $M+$ and then unload.

Jacobian corresponding to (34) is given by

$$
J=\left[\begin{array}{cccccc}
\frac{\partial e\left(t^{1}\right)}{\partial \alpha_{1,1}} & \cdots & \frac{\partial e\left(t^{1}\right)}{\partial \alpha_{K_{\alpha}}, M_{\alpha}} & \frac{\partial e\left(t^{1}\right)}{\partial \beta_{1}} & \ldots & \frac{\partial e\left(t^{1}\right)}{\partial \beta_{K_{\beta}}} \\
\vdots & \ddots & \vdots & \vdots & \ddots & \vdots \\
\frac{\partial e\left(t^{N_{k}}\right)}{\partial \alpha_{1,1}} & \cdots & \frac{\partial e\left(t^{N_{k}}\right)}{\partial \alpha_{K_{\alpha}, M_{\alpha}}} & \frac{\partial e\left(t^{N_{k}}\right)}{\partial \beta_{1}} & \cdots & \frac{\partial e\left(t^{N_{k}}\right)}{\partial \beta_{K_{\beta}}}
\end{array}\right] .
$$

where

$$
\begin{gathered}
\frac{\partial e\left(t^{k}\right)}{\partial \alpha_{m}}=\sum_{i=1}^{N_{i}} \sum_{j=1}^{N_{j}}\left(-\phi_{m}\left(\sigma_{R_{i}}\right) \nu_{I}\left(\sigma_{I_{j}}\right) \bar{\varepsilon}\left(\sigma\left(t^{k}\right)+\sigma_{I_{j}}, T\left(t^{k}\right), \sigma_{R_{i}}\right) w_{i} w_{j}+\frac{1}{\sum_{l} \alpha_{l}} \varepsilon\left(\sigma\left(t^{k}\right), T\left(t^{k}\right)\right)\right) \\
\frac{\partial e\left(t^{k}\right)}{\partial \beta_{m}}=\sum_{i=1}^{N_{i}} \sum_{j=1}^{N_{j}}\left(-\nu_{R}\left(\sigma_{R_{i}}\right) \psi_{m}\left(\sigma_{I_{j}}\right) \bar{\varepsilon}\left(\sigma\left(t^{k}\right)+\sigma_{I_{j}}, T\left(t^{k}\right), \sigma_{R_{i}}\right) w_{i} w_{j}+\frac{1}{\sum_{l} \beta_{l}} \varepsilon\left(\sigma\left(t^{k}\right), T\left(t^{k}\right)\right)\right) .
\end{gathered}
$$

\subsection{Densities and Model Parameters}

Optimizing the densities and model parameters involves two minimization routines. In the first step, the sum of squared errors

$$
F\left(\vec{q}_{\varepsilon}\right)=\frac{1}{2} \sum_{k=1}^{N_{k}} e_{\varepsilon}\left(t^{k}\right)^{2}=\frac{1}{2} \sum_{k=1}^{N_{k}}\left(\hat{\varepsilon}^{k}-\varepsilon\left(\sigma\left(t^{k}\right), T\left(t^{k}\right)\right)\right)^{2}
$$

between the measured strain and predicted strain is minimized. Here the design variables are

$$
\vec{q}_{\varepsilon}=\left[\alpha_{1,1}, \cdots, \alpha_{K_{\alpha}, M_{\alpha}}, \beta_{1}, \cdots, \beta_{K_{\beta}}, E_{A}, E_{M}, \sigma_{L}, \Delta \sigma_{T}, \varepsilon_{T}, \tau, V\right]^{T} .
$$

Note that we do not include the transition temperature $T_{L}$ due to its coupling with $\sigma_{L}$. The determination of only one of the two is necessary.

In the second step, the sum of squared errors

$$
F\left(\vec{q}_{\Omega}\right)=\frac{1}{2} \sum_{k=1}^{N_{k}} e_{\Omega}\left(t^{k}\right)^{2}=\frac{1}{2} \sum_{k=1}^{N_{k}}\left(\hat{\Omega}^{k}-\Omega\left(\sigma\left(t^{k}\right), T\left(t^{k}\right)\right)\right)^{2}
$$

between the measured resistance $\hat{\Omega}^{k}$ and the predicted resistance $\Omega\left(\sigma\left(t^{k}\right), T\left(t^{k}\right)\right)$ is minimized. The design variables in this step are

$$
\vec{q}_{\Omega}=\left[\rho_{0 A}, \rho_{0 M}, \alpha_{A}, \alpha_{M}, \nu\right]^{T} .
$$


Again, the analytical Jacobian is derived to decrease computation time. The partial derivatives corresponding to (37) and (38) are given

$$
\begin{aligned}
& \frac{\partial e_{\varepsilon}\left(t^{k}\right)}{\partial \alpha_{m}}: \text { See Equation (35) } \\
& \frac{\partial e_{\varepsilon}\left(t^{k}\right)}{\partial \beta_{m}}: \text { See Equation (36) } \\
& \frac{\partial e_{\varepsilon}\left(t^{k}\right)}{\partial E_{A}}=\frac{x_{A}\left(t^{k}\right) \sigma\left(t^{k}\right)}{E_{A}^{2}} \\
& \frac{\partial e_{\varepsilon}\left(t^{k}\right)}{\partial E_{M}}=\sigma\left(t_{k}\right) \frac{x_{M+}\left(t^{k}\right)+x_{M-}\left(t^{k}\right)}{E_{M}^{2}} \\
& \frac{\partial e_{\varepsilon}\left(t^{k}\right)}{\partial \sigma_{L}}=-\frac{\partial x_{M+}}{\partial \sigma_{L}}\left(\frac{\sigma\left(t^{k}\right)}{E_{M}}-\frac{\sigma\left(t^{k}\right)}{E_{A}}+\varepsilon_{T}\right)-\frac{\partial x_{M-}}{\partial \sigma_{L}}\left(\frac{\sigma\left(t^{k}\right)}{E_{M}}-\frac{\sigma\left(t^{k}\right)}{E_{A}}-\varepsilon_{T}\right) \\
& \frac{\partial e_{\varepsilon}\left(t^{k}\right)}{\partial \Delta \sigma_{T}}=-\frac{\partial x_{M+}}{\partial \Delta \sigma_{T}}\left(\frac{\sigma\left(t^{k}\right)}{E_{M}}-\frac{\sigma\left(t^{k}\right)}{E_{A}}+\varepsilon_{T}\right)-\frac{\partial x_{M-}}{\partial \Delta \sigma_{T}}\left(\frac{\sigma\left(t^{k}\right)}{E_{M}}-\frac{\sigma\left(t^{k}\right)}{E_{A}}-\varepsilon_{T}\right) \\
& \frac{\partial e_{\varepsilon}\left(t^{k}\right)}{\partial \varepsilon_{T}}=x_{M+}\left(t^{k}\right)-x_{M-}\left(t^{k}\right) . \\
& \frac{\partial e_{\varepsilon}\left(t^{k}\right)}{\partial \tau_{x}}=-\frac{\partial x_{M+}}{\partial \tau}\left(\frac{\sigma\left(t^{k}\right)}{E_{M}}-\frac{\sigma\left(t^{k}\right)}{E_{A}}+\varepsilon_{T}\right)-\frac{\partial x_{M-}}{\partial \tau}\left(\frac{\sigma\left(t^{k}\right)}{E_{M}}-\frac{\sigma\left(t^{k}\right)}{E_{A}}-\varepsilon_{T}\right) \\
& \frac{\partial e_{\varepsilon}\left(t^{k}\right)}{\partial V}=-\frac{\partial x_{M+}}{\partial V}\left(\frac{\sigma\left(t^{k}\right)}{E_{M}}-\frac{\sigma\left(t^{k}\right)}{E_{A}}+\varepsilon_{T}\right)-\frac{\partial x_{M-}}{\partial V}\left(\frac{\sigma\left(t^{k}\right)}{E_{M}}-\frac{\sigma\left(t^{k}\right)}{E_{A}}-\varepsilon_{T}\right) \\
& \frac{\partial e_{\Omega}\left(t^{k}\right)}{\partial \rho_{0 A}}=-\frac{L\left(\varepsilon_{e}\right)}{A\left(\varepsilon\left(t^{k}\right)\right)} x_{A}\left(t^{k}\right)\left(1+\alpha_{A} \Delta T\left(t^{k}\right)\right) \\
& \frac{\partial e_{\Omega}\left(t^{k}\right)}{\partial \rho_{0 M}}=-\frac{L\left(\varepsilon_{e}\right)}{A\left(\varepsilon\left(t^{k}\right)\right)}\left(x_{M+}\left(t^{k}\right)+x_{M-}\left(t^{k}\right)\right)\left(1+\alpha_{M} \Delta T\left(t^{k}\right)\right) \\
& \frac{\partial e_{\Omega}\left(t^{k}\right)}{\partial \alpha_{A}}=-\frac{L\left(\varepsilon_{e}\right)}{A\left(\varepsilon\left(t^{k}\right)\right)} x_{A}\left(t^{k}\right) \rho_{0 A} \Delta T \\
& \frac{\partial e_{\Omega}\left(t^{k}\right)}{\partial \alpha_{M}}=-\frac{L\left(\varepsilon_{e}\right)}{A\left(\varepsilon\left(t^{k}\right)\right)}\left(x_{M+}\left(t^{k}\right)+x_{M-}\left(t^{k}\right)\right) \rho_{0 M} \Delta T\left(t^{k}\right) \\
& \frac{\partial e_{\Omega}\left(t^{k}\right)}{\partial \nu}=-\frac{2 L\left(\varepsilon_{e}\right) \rho_{s m a}\left(t^{k}\right) \varepsilon_{e}\left(t^{k}\right)}{\pi R_{0}^{2}\left(1-\nu \varepsilon_{e}\left(t^{k}\right)\right)^{3}}
\end{aligned}
$$

The partial derivatives of the phase fractions with respect to $\sigma_{L}, \Delta \sigma_{T}, \tau$, and $V$ are found by differentiating the phase fraction evolution ODEs (4) and switching the order of the derivative, yielding

$$
\begin{aligned}
& \frac{d}{d t} \frac{\partial x_{M+}(t)}{\partial \gamma}=-\frac{\partial p_{+A}}{\partial \gamma} x_{M+}(t)-p_{+A} \frac{\partial x_{M+}(t)}{\partial \gamma}+\frac{\partial p_{A+}}{\partial \gamma} x_{A}(t)-p_{A+}\left(\frac{\partial x_{M+}(t)}{\partial \gamma}+\frac{\partial x_{M-}(t)}{\partial \gamma}\right) \\
& \frac{d}{d t} \frac{\partial x_{M-}(t)}{\partial \gamma}=-\frac{\partial p_{-A}}{\partial \gamma} x_{M-}(t)-p_{+A} \frac{\partial x_{M-}(t)}{\partial \gamma}+\frac{\partial p_{A-}}{\partial \gamma} x_{A}(t)-p_{A-}\left(\frac{\partial x_{M+}(t)}{\partial \gamma}+\frac{\partial x_{M-}(t)}{\partial \gamma}\right) .
\end{aligned}
$$

where $\gamma=\sigma_{L}, \Delta \sigma_{T}, \tau$, and $V$. The derivatives of the transition rates are

$$
\frac{\partial p_{\alpha \beta}}{\partial \tau}=-\frac{1}{\tau} p_{\alpha \beta},
$$




$$
\begin{gathered}
\frac{\partial p_{\alpha \beta}}{\partial V}=-\frac{\Delta G_{\alpha \beta}}{k_{b} T(t)} p_{\alpha \beta}, \\
\frac{\partial p_{\alpha \beta}}{\partial \sigma_{L}}=-\frac{V}{k_{b} T(t)} p_{\alpha \beta} \frac{\partial \Delta G_{\alpha \beta}}{\partial \sigma_{L}},
\end{gathered}
$$

and

$$
\frac{\partial p_{\alpha \beta}}{\partial \Delta \sigma_{T}}=-\frac{V}{k_{b} T(t)} p_{\alpha \beta} \frac{\partial \Delta G_{\alpha \beta}}{\partial \Delta \sigma_{T}} .
$$

The Gibbs energy derivatives $\frac{\partial \Delta G_{\alpha \beta}}{\partial \sigma_{L}}$ and $\frac{\partial \Delta G_{\alpha \beta}}{\partial \Delta \sigma_{T}}$ are found by differentiating (1). Finally, the partial derivatives of the phase fractions are found by integrating (53).

\section{$5 \quad$ Experimental Setup}

As shown in Figure 16, the SMA experiments were conducted in a temperature-controlled water bath to ensure constant temperature conditions. The water temperature was regulated using a heating element. A low-temperature FLEXINOL wire $\left(T_{A_{s}}=343 \mathrm{~K}\right.$, diameter $=0.005$ in. $(0.127 \mathrm{~mm})$, Dynalloy Inc., Tustin, CA) was used. The displacement of one end of the wire was controlled with a Micro-Drives motor and BOP 20-10m operational power supply (Kepco Inc., Flushing, NY) and measured with a mDMS-D170 infrared displacement sensor (Philtec Inc., Annapolis, MD). For resistance measurements, leads were connected to the wire and a small current (50 mA) was applied using a 6454A operational power supply (Agilent Technologies, Santa Clara, CA). The voltage was monitored in order to determine the resistance, and the force in the SMA wire was measured using a MLP-10 load cell (Transducer Techniques, Temecula, CA).

The experiments were conducted over a range of temperatures: $308,318,328,338,343,348,353$, and $358 \mathrm{~K}$. The zero-strain lengths in the wire were determined from its austenitic length. At temperatures above $343 \mathrm{~K}$ (the austenitic start temperature), the zero-strain length corresponds to the point where a force in the wire is initially detected. The length at $343 \mathrm{~K}$ is used for all tests below this temperature, as the wire can exist in a blend of all three phases below the austenitic start temperature due to material inhomogeneities. A specific procedure described below was followed for each test to ensure a known initial condition.

1. Heat the wire to $358 \mathrm{~K}$ and strain it to $3.5 \%$ strain. Apply $0.6 \mathrm{~A}$ current and measure the force $\left(F_{0}\right)$.

2. Set the desired water temperature.

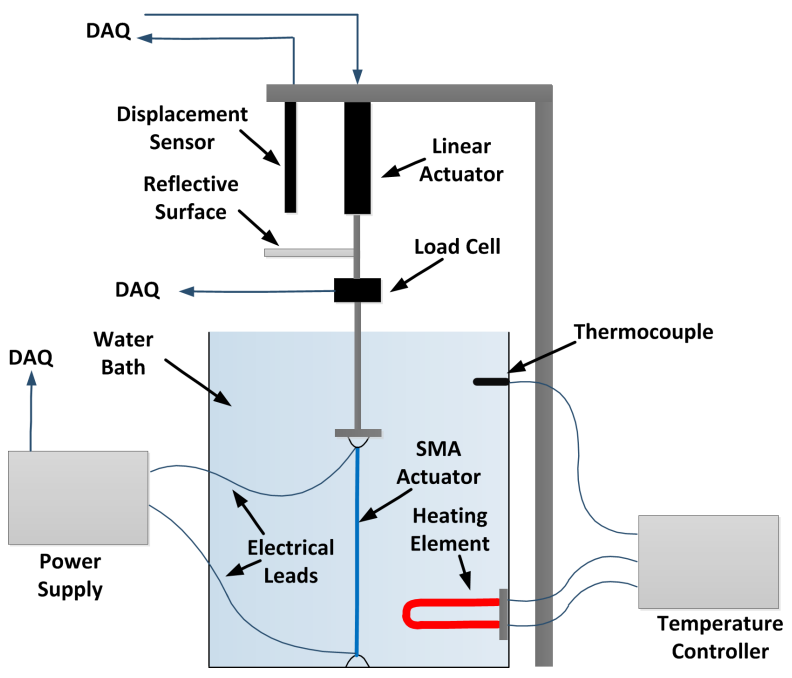

Figure 16: Experimental setup. 
3. Displace the wire to $3.5 \%$. Increase the current in the wire using the Agilent power supply until the force equals the force achieved in the $358 \mathrm{~K}$ wire at $3.5 \%$ and $0.6 \mathrm{~A}\left(F_{0}\right)$. Maintain this current while the displacement is adjusted to the zero-strain length.

4. Set the current to zero and let the wire cool for 3 minutes while the water is stirred to reach equilibrium.

5. Strain the wire to $7.0 \%$ and back to $0.0 \%$.

6. Repeat steps $2-5$ three times.

7. Go to the next temperature, starting with step 2 .

\section{Model Validation Results}

\subsection{Estimation of Model Parameters}

Initial estimates of the model parameters are obtained using the techniques described in Section 3. The experimental stress-strain data at two different temperatures is shown in Figure 17(a) and the creep data at two different strain values is shown in Figure 17(b). The resistance-strain data used to estimate $\rho_{M 0}$ and $\nu$ is shown in Figure 17(c), and the data used to estimate $\rho_{A 0}$ is shown in Figure 17(d). The resistance-strain data at two temperatures is shown in Figure 17(e) and used to estimate $\alpha_{M}$. An initial estimate for $\alpha_{A}$ could not be obtained due to a decrease in resistance as temperature increased, as shown in Figure 17(f). This phenomenon is likely due to more of the wire converting to austenite as the temperature increases and the fact that the nominal resistivity of austenite is lower than martensite. Therefore, the estimate for $\alpha_{M}$ is used for $\alpha_{A}$. The initial estimates for all the model parameters are listed in Table 6 .

Two of the parameter estimates listed in Table 6 deviate from expected and published values. The austenitic elastic modulus is considerably lower than other published values (70 GPa). The lower value may be due to compliance in the experimental setup or slipping of the wire. Additionally, the Poisson's ratio is approximately twice the value expected for the material. This may be due to error in measuring resistance or the power supply leads being affected during loading.

Initial estimates for the relative and interaction stress densities are found by determining the experimental mean and standard deviations for the relative stress, $148.1 \mathrm{MPa}$ and $30.5 \mathrm{MPa}$, respectively, and standard deviation for the interaction stress, 35.4 MPa. The relative stress parameters are then converted to lognormal form using (32) and (33). This method is used because it was found that it produces a better fit versus estimating the parameters using the $95.4 \%$ confidence intervals (Figures 13 and 14). The relative stress and interaction stress densities at $353 \mathrm{~K}$ are shown in Figure 18.

\subsection{Densities Only}

The parameters for the density basis functions (13) and (14) are chosen a priori using the initial estimates for the relative stress mean and standard deviation. Three different values for the mean $\mu_{R_{m}}$ are used: $\mathrm{E}\left(\hat{\sigma}_{R}^{k}\right)$ and $\mathrm{E}\left(\hat{\sigma}_{R}^{k}\right) \pm 75 \mathrm{MPa}$. Two different values for the standard deviation $c_{k}$ are used: the initial estimate (30.5 $\mathrm{MPa})$ and five times this value (152.5 MPa). A combination of these values produces six basis functions for the relative stress density $\nu_{R}\left(\sigma_{R}\right)$. The basis functions for the interaction stress density are parameterized with five different values: $0.25 b, 0.5 b, b, 2 b$, and $4 b$.

The stress-strain data at 338 and $353 \mathrm{~K}$ is used to optimize the density coefficients $\alpha_{k, m}$ and $\beta_{k}$. The experimental data at $338 \mathrm{~K}$ is used instead of the lowest temperature data $(308 \mathrm{~K})$ due to the two-way shape memory effect seen in the FLEXINOL wires [5]. Since the model does not currently quantify this effect, the experimental data immediately below the austenitic start temperature is used.

Comparisons between the experimental stress-strain data and the model are shown in Figure 19, and comparisons between the experimental resistance-strain data and the model are shown in Figure 20. The fit model $(338$ and $353 \mathrm{~K})$ is shown in Figures 19(a) and (b). All other results $(308,328,343$, and $348 \mathrm{~K})$ are 
predicted model results, including all the resistance-strain plots in Figure 20. The final cost (sum of squared error) for the fit model is 0.0422 (or a mean squared error of $3.01 \times 10^{-5}$ ).

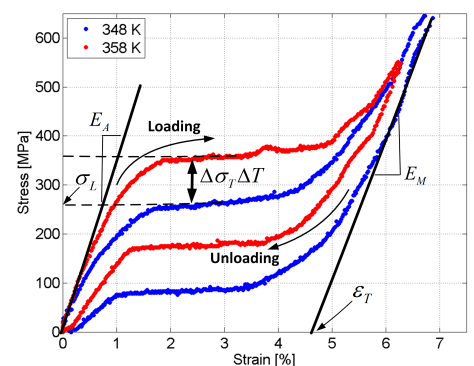

(a)

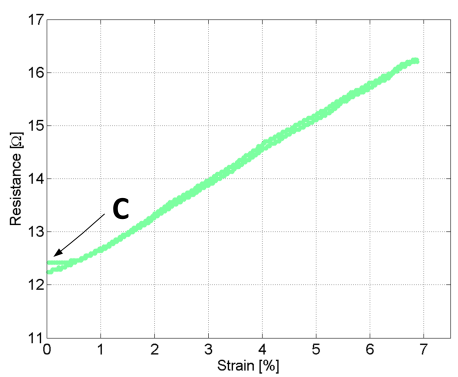

(d)

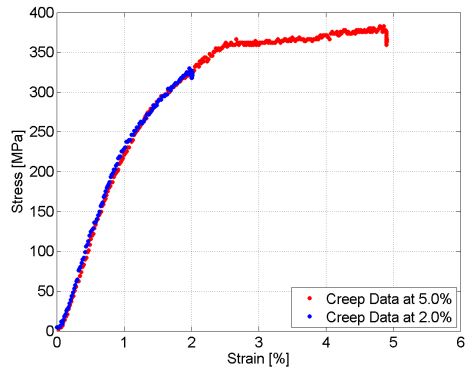

(b)

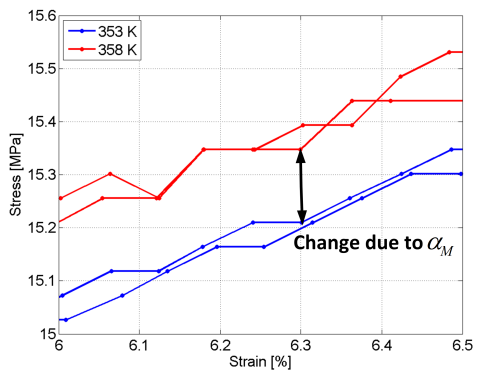

(e)

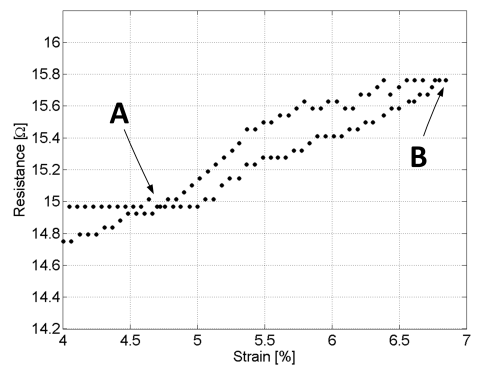

(c)

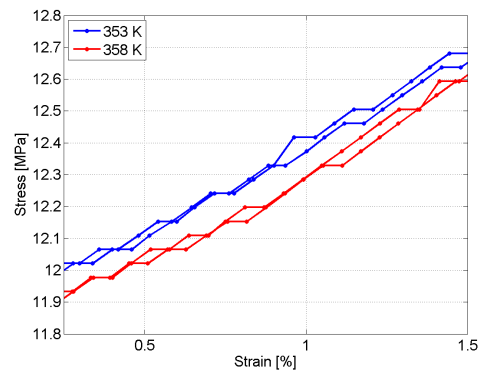

(f)

Figure 17: Identification of model parameters: (a) stress-strain data at $348 \mathrm{~K}$ and $358 \mathrm{~K}$; (b) creep data at $5.0 \%$ and $2.0 \%$ strain; (c) low-temperature resistance-strain data for estimating $\rho_{M 0}$ (point $A$ ) and $\nu$ (change over $A$ to $B$ ); (d) high-temperature resistance-strain data for estimating $\rho_{A 0}$ (point $C$ ); (e) resistance-strain data at two temperatures for estimating $\alpha_{M}$; (f) resistance-strain data at two temperatures in the austenitic regime.

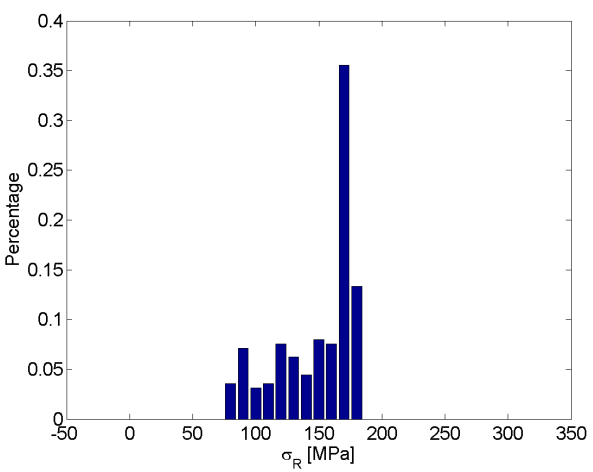

(a)

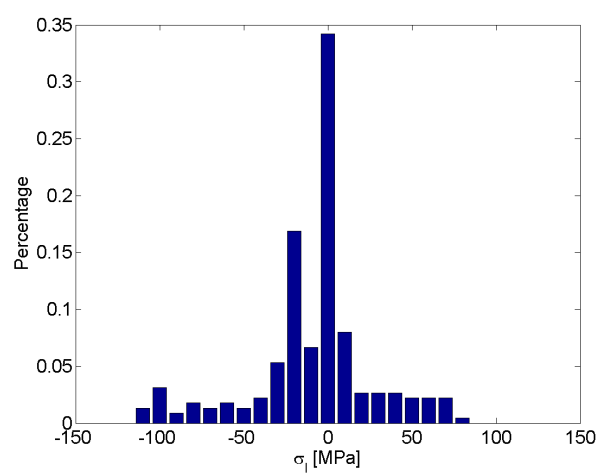

(b)

Figure 18: Identification of density parameters: (a) distribution of experimental relative stress; (b) distribution of experimental interaction stress. 


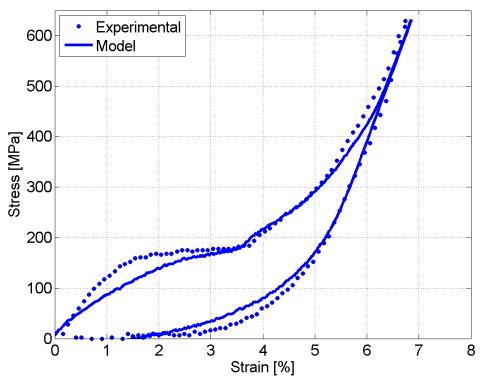

(a)

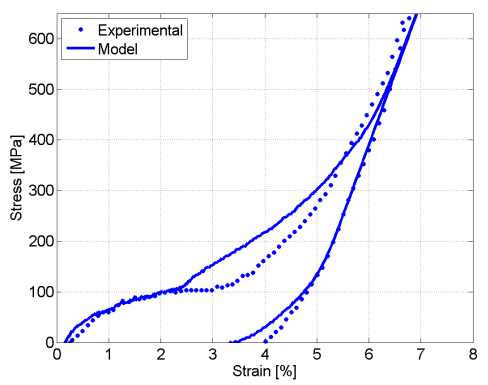

(d)

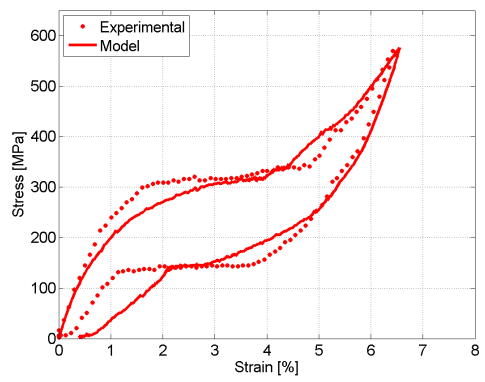

(b)

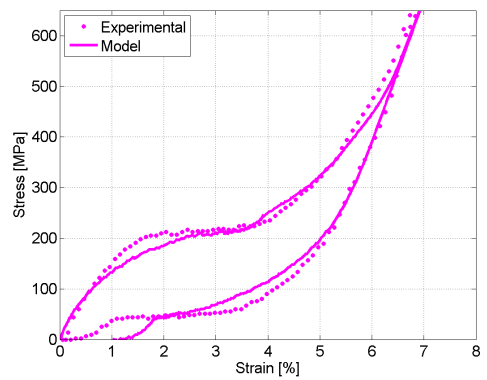

(e)

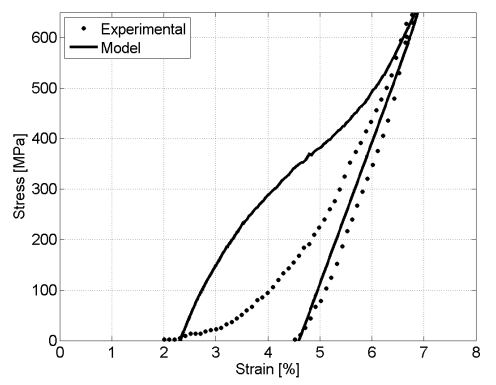

(c)

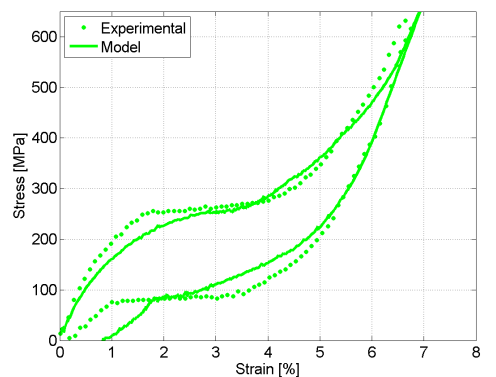

(f)

Figure 19: Stress versus strain results for optimized densities and model parameters: Comparison between model fit and experimental data at (a) $338 \mathrm{~K}$ and (b) $353 \mathrm{~K}$; Comparison between model prediction and experimental data at (c) $308 \mathrm{~K}$, (d) $328 \mathrm{~K}$, (e) $343 \mathrm{~K}$, and (f) $348 \mathrm{~K}$.

Figures 19 and 20 reveal a number of interesting results. As expected, the model fails to accurately quantify the lowest temperature $(308 \mathrm{~K})$ response shown in Figure 19(c), where the material is undergoing a transition from a blend of $M+/ M-$ to $M+$. The model predicts a much higher transition stress than observed in experimental data. While the data is not used in the optimization algorithm, it is worth noting, that the lowest temperature data has the highest mean squared error (MSE): $2.72 \times 10^{-4}$. The other predicted model results $\left(328,343\right.$, and $348 \mathrm{~K}$ ) have a MSE on the order of the fit model: $5.23 \times 10^{-5}, 2.24 \times 10^{-5}$, and $2.71 \times 10^{-5}$, respectively. These results indicate the model is accurately quantifying the material behavior without over-fitting the data.

The resistance-strain data in Figure 20 shows that the model matches the experimental data best at high temperatures; see Figures 20(b), (c), and (f). As actuators operate at higher temperatures (in order to recover strain above $T_{A_{s}}$ ) the results indicate that the model may be suitable for self-sensing applications, where the resistance in the wire is used to predict strain $[7,8]$. However, operating in low temperatures may be problematic for these applications, as multiple strain values correspond to a single resistance measurement (strains below 2\% in Figures 20(c) and (d)).

The optimal density coefficients are listed in Tables 4 and 5, and the corresponding densities are shown in Figure 21. The relative stress density is shown in Figure 21(a), and the interaction stress density is shown in Figure 21(b). The optimal density coefficients reveal that the polycrystalline behavior is largely modeled using the interaction stress. The highest coefficient is on the largest standard deviation (141.60 MPa). The model averages the transition behavior without exactly quantifying the transition stresses. As shown in the high temperature stress-strain results (Figures 19(b), (e), and (f)), the model matches the midpoints of the loading and unloading curves, but fails to quantify the initial and final transition points (around 1\% and $4 \%$ strain in the figures). This discrepancy is likely due to errors in the initial estimates of the other model 


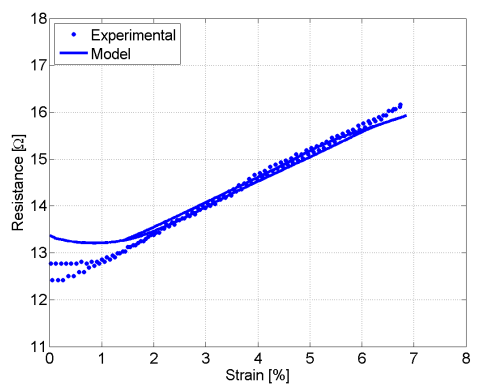

(a)

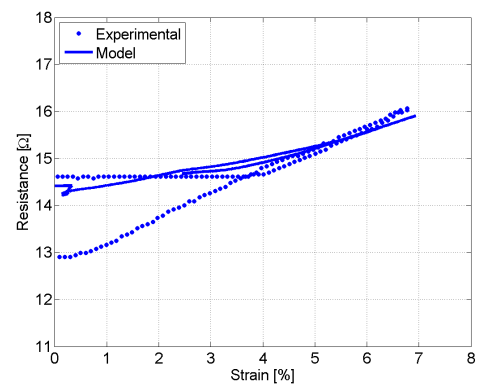

(d)

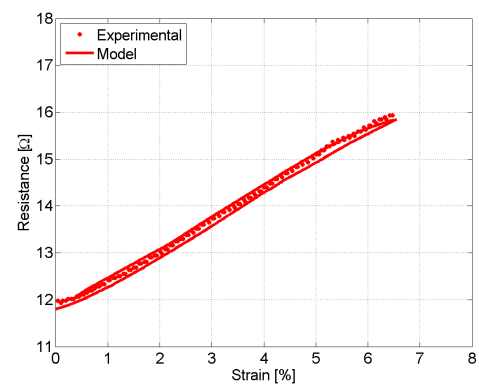

(b)

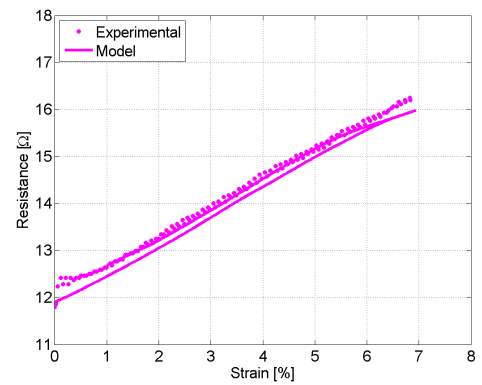

(e)

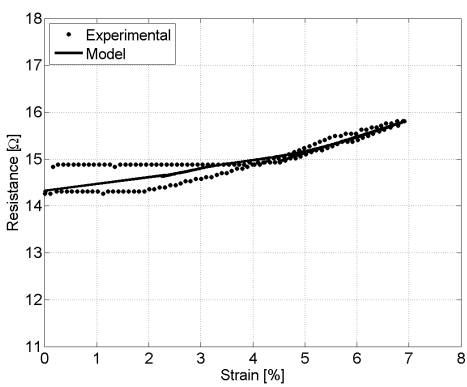

(c)

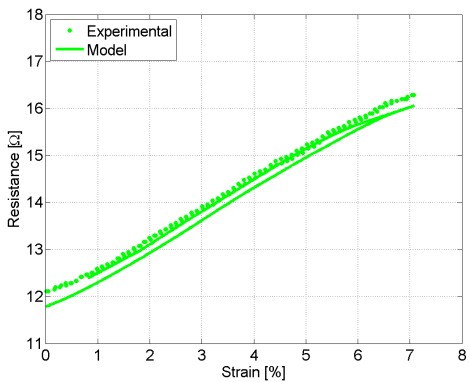

(f)

Figure 20: Resistance versus strain results for optimized densities: Comparison between model fit and experimental data at (a) $338 \mathrm{~K}$ and (b) $353 \mathrm{~K}$; Comparison between model prediction and experimental data at (c) $308 \mathrm{~K}$, (d) $328 \mathrm{~K}$, (e) $343 \mathrm{~K}$, and (f) $348 \mathrm{~K}$.

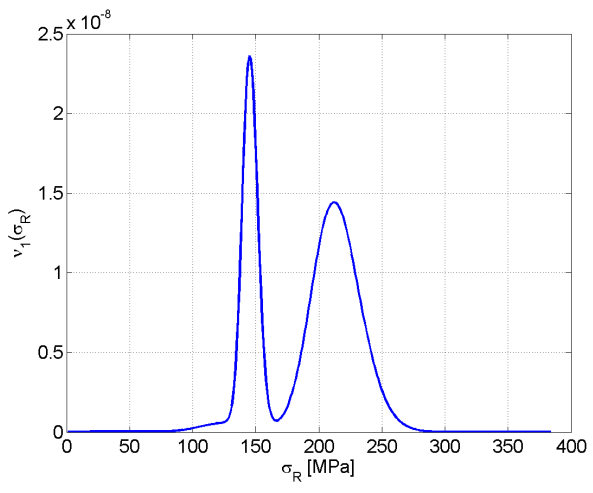

(a)

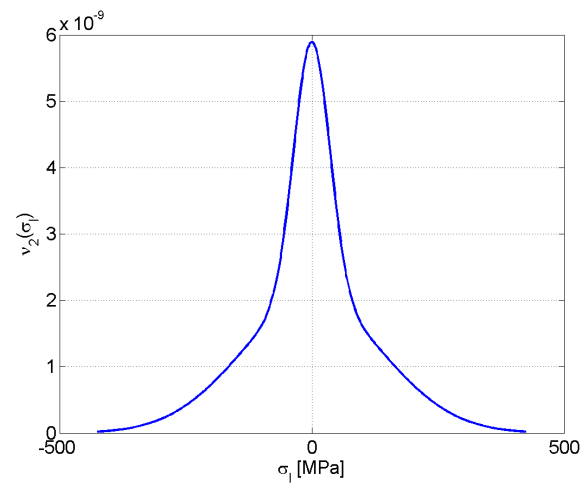

(b)

Figure 21: Optimized densities: (a) relative stress density $\nu_{R}\left(\sigma_{R}\right)$; (b) interaction stress density $\nu_{I}\left(\sigma_{I}\right)$.

parameters, which motivates the need for optimizing these estimates.

The kernel number does not affect the computational efficiency of the model, since the densities and quadrature weights can be pre-computed once the optimal densities are known; however, the optimal coefficients listed in Tables 4 and 5 reveal that some of the kernels are unnecessary. The kernels with coefficients 
Table 4: Optimized coefficients and parameters for $\nu_{R}\left(\sigma_{R}\right)$ for optimization of the densities only.

\begin{tabular}{ccccc}
\hline \hline$m$ & $k$ & $\alpha_{k, m}$ & $\mu_{R_{m}}$ & $c_{k}$ \\
\hline 1 & 1 & 0.000 & 18.03 & 0.159 \\
1 & 2 & 0.004 & 17.80 & 0.621 \\
2 & 1 & 0.324 & 18.79 & 0.041 \\
2 & 2 & 0.035 & 18.72 & 0.191 \\
3 & 1 & 0.000 & 19.22 & 0.019 \\
3 & 2 & 0.636 & 19.18 & 0.089 \\
\hline
\end{tabular}

Table 5: Optimized coefficients and parameters for $\nu_{I}\left(\sigma_{I}\right)$ for optimization of the densities only.

\begin{tabular}{ccc}
\hline \hline$k$ & $\beta_{k}$ & $b_{k}(\mathrm{MPa})$ \\
\hline 1 & 0.000 & 8.85 \\
2 & 0.000 & 17.70 \\
3 & 0.329 & 35.40 \\
4 & 0.002 & 70.80 \\
5 & 0.669 & 141.60 \\
\hline
\end{tabular}

less than 0.005 can be neglected. Alternatively, the kernels with large optimal coefficients can be perturbed to create new kernels, and the optimization algorithm can be run again.

\subsection{Densities and Model Parameters}

For the optimization of the densities and model parameters, the same density basis functions are used; the values for $\mu_{R_{m}}$ and $c_{k}$ listed in Table 4 and the $b_{k}$ values listed in Table 5 . Since the strain model and resistance model parameters are optimized, both the stress-strain data and resistance-strain data at $338 \mathrm{~K}$ and $353 \mathrm{~K}$ are used. The stress-strain model parameters and densities are optimized first. Using the optimal values for the strain model, the resistance model parameters are optimized next.

Comparisons between the model and experimental data are shown in Figures 22 and 23. The fit model (338 and $353 \mathrm{~K}$ ) is shown in Figures 22(a) and (b) and Figures 23(a) and (b). The other figures compare the predicted model to the experimental data. The final sum of squared errors for the stress-strain optimization is 0.0060 (or a MSE of $4.29 \times 10^{-6}$ ). The sum of squared errors for the optimization of the densities and model parameters is $85 \%$ lower than optimization of the densities only. A comparison of Figures 19(a) and (b) and Figures 22 (a) and (b) reveals that optimizing the model parameters allows the model to accurately capture the initial and final transition regions from austenite to martensite, instead of averaging the response.

Even optimizing the model parameters fails to quantify the low-temperature stress-strain response shown in Figure 22(d). The MSE for the $308 \mathrm{~K}$ data is $2.55 \times 10^{-4}$, similar to the results for optimizing densities only. The predicted model MSEs compare favorably to the optimization of densities only: $9.53 \times 10^{-5}, 1.56 \times 10^{-5}$, and $2.22 \times 10^{-5}$ for 328,343 , and $343 \mathrm{~K}$, respectively. Whereas the fit model MSE is significantly lower for the optimization of densities and model parameters, the predicted model MSEs are the same order of magnitude as the optimization of densities only, suggesting that the initial estimates for the model parameters are accurate. The optimized model parameters are listed in Table 6 along with the initial estimates.

The optimal density coefficients are listed in Tables 7 and 8. The corresponding densities are shown in Figures 24(a) and (b). Comparing these densities to the previous ones (Figure 21) indicates that the standard deviations for the overall densities are lower, especially for the interaction stress density. Optimizing all the parameters reduces the reliance on the interaction stress density for modeling the polycrystalline behavior. 


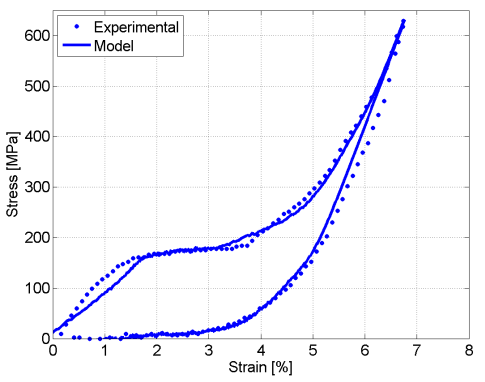

(a)

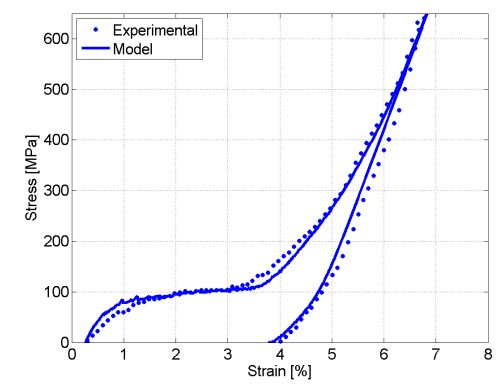

(d)

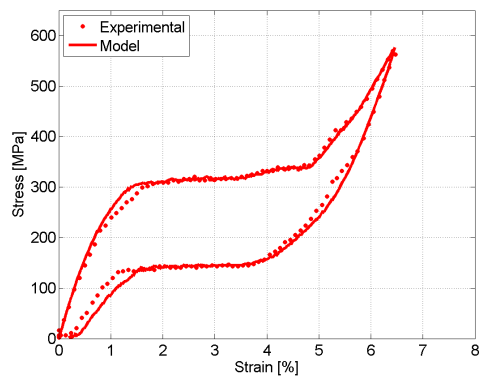

(b)

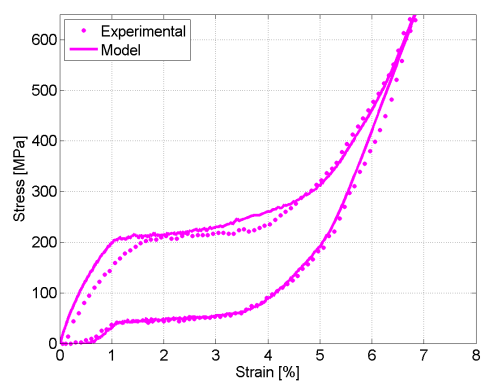

(e)

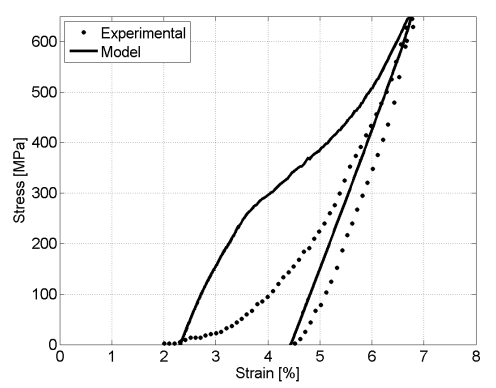

(c)

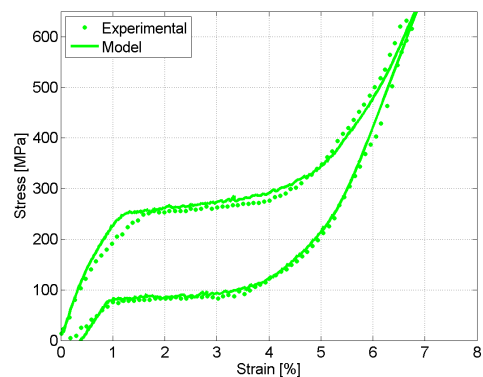

(f)

Figure 22: Stress versus strain results for optimized densities and model parameters: Comparison between model fit and experimental data at (a) $338 \mathrm{~K}$ and (b) $353 \mathrm{~K}$; Comparison between model prediction and experimental data at (c) $308 \mathrm{~K}$, (d) $328 \mathrm{~K}$, (e) $343 \mathrm{~K}$, and (f) $348 \mathrm{~K}$.

Table 6: Comparison between initial estimates of SMA model parameters and optimal values.

\begin{tabular}{cccc}
\hline \hline Variable & Initial estimate & Optimal value & Units \\
\hline$E_{A}$ & 35.0 & 35.0 & $\mathrm{GPa}$ \\
$E_{M}$ & 28.0 & 27.2 & $\mathrm{GPa}$ \\
$\sigma_{L}$ & 275 & 295 & $\mathrm{MPa}$ \\
$\Delta \sigma_{T}$ & 10 & 9.2 & $\mathrm{MPa} / \mathrm{K}$ \\
$\varepsilon_{T}$ & 4.6 & 4.44 & $\%$ \\
$\tau$ & 1.09 & 1.78 & $\mathrm{~s}$ \\
$V$ & $8.0 \times 10^{-26}$ & $9.5 \times 10^{-26}$ & $\mathrm{~m}^{3}$ \\
$\rho_{0 A}$ & $8.8 \times 10^{-7}$ & $8.9 \times 10^{-7}$ & $\mathrm{Ohm}-\mathrm{m}$ \\
$\rho_{0 M}$ & $10.7 \times 10^{-7}$ & $10.7 \times 10^{-7}$ & $\mathrm{Ohm}-\mathrm{m}$ \\
$\alpha_{A}$ & 0.0003 & 0.0001 & $\mathrm{Ohm}-\mathrm{m} / \mathrm{K}$ \\
$\alpha_{M}$ & 0.0003 & 0.0001 & $\mathrm{Ohm}-\mathrm{m} / \mathrm{K}$ \\
$\nu$ & 0.67 & 0.8 & - \\
\hline
\end{tabular}

It is worth noting that even optimizing the model on the $308 \mathrm{~K}$ data fails to quantify the behavior observed experimentally. Using the low-temperature data in the optimization algorithm produces a MSE of 


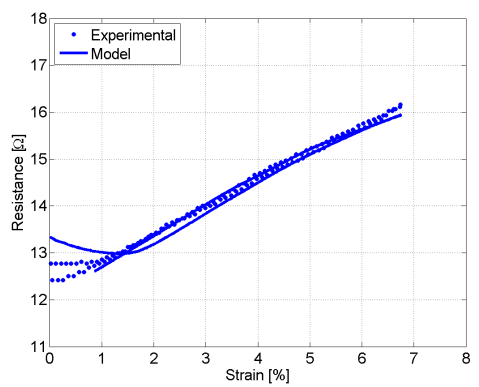

(a)

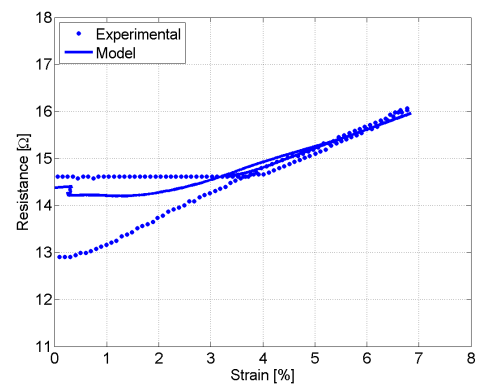

(d)

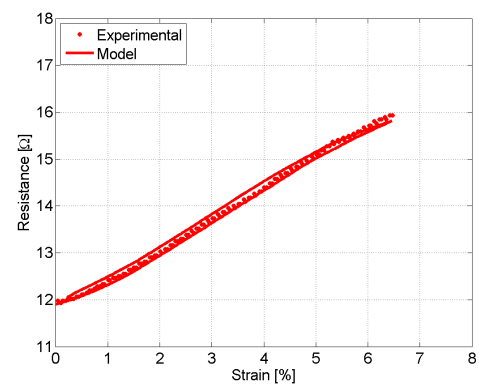

(b)

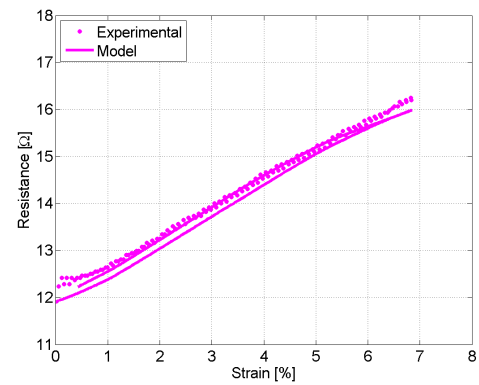

(e)

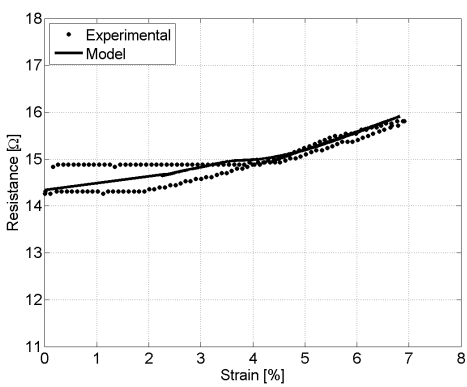

(c)

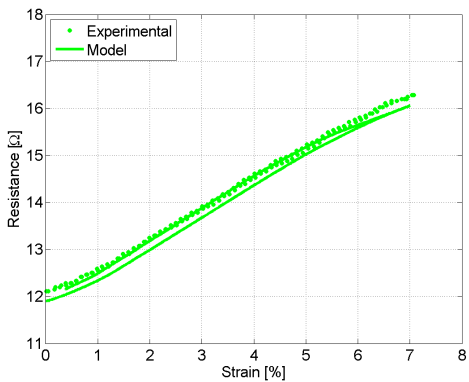

(f)

Figure 23: Resistance versus strain results for optimized densities and model parameters: Comparison between model fit and experimental data at (a) $338 \mathrm{~K}$ and (b) $353 \mathrm{~K}$; Comparison between model prediction and experimental data at (c) $308 \mathrm{~K}$, (d) $328 \mathrm{~K}$, (e) $343 \mathrm{~K}$, and (f) $348 \mathrm{~K}$.

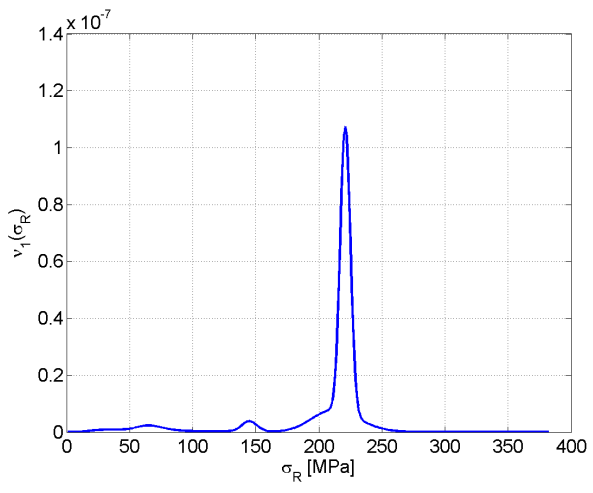

(a)

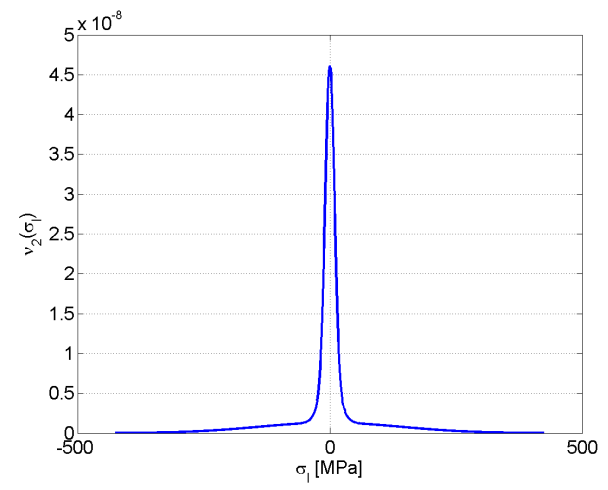

(b)

Figure 24: Optimized densities: (a) relative stress density $\nu_{1}\left(\sigma_{R}\right)$; (b) interaction stress density $\nu_{2}\left(\sigma_{I}\right)$.

$1.62 \times 10^{-4}$. This value is lower than the $308 \mathrm{~K}$ MSE when the optimization uses the 338 and $353 \mathrm{~K}$ data, but it is still an order of magnitude higher than the high-temperature data. Additionally, the MSEs for the other temperatures increase. 
Table 7: Optimized coefficients and parameters for $\nu_{R}\left(\sigma_{R}\right)$ for optimization of the densities and model parameters.

\begin{tabular}{ccccc}
\hline \hline$m$ & $k$ & $\alpha_{k, m}$ & $\mu_{R_{m}}$ & $c_{k}$ \\
\hline 1 & 1 & 0.010 & 18.03 & 0.159 \\
1 & 2 & 0.019 & 17.80 & 0.621 \\
2 & 1 & 0.306 & 18.79 & 0.041 \\
2 & 2 & 0.053 & 18.72 & 0.191 \\
3 & 1 & 0.271 & 19.22 & 0.019 \\
3 & 2 & 0.340 & 19.18 & 0.089 \\
\hline
\end{tabular}

Table 8: Optimized coefficients and parameters for $\nu_{I}\left(\sigma_{I}\right)$ for optimization of the densities and model parameters.

\begin{tabular}{ccc}
\hline \hline$k$ & $\beta_{k}$ & $b_{k}[\mathrm{MPa}]$ \\
\hline 1 & 0.004 & 8.85 \\
2 & 0.031 & 17.70 \\
3 & 0.249 & 35.40 \\
4 & 0.075 & 70.80 \\
5 & 0.641 & 141.60 \\
\hline
\end{tabular}

\subsection{Minor Loop Comparison}

Minor loop data was obtained on the experimental setup at $353 \mathrm{~K}$ on a different SMA wire. The data is collected by reversing the direction of stress within the transition region identified in Figure 13, either on loading or unloading. Example experimental data that quantifies a minor loop during both loading and unloading is shown in Figure 25.

Since the minor loop data was obtained on a different wire, the strain model parameters (including the densities) are optimized again, resulting in the optimal parameters listed in Table 9. The optimal density

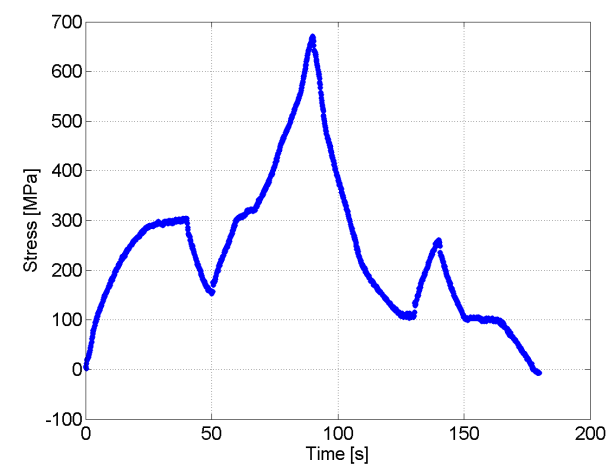

(a)

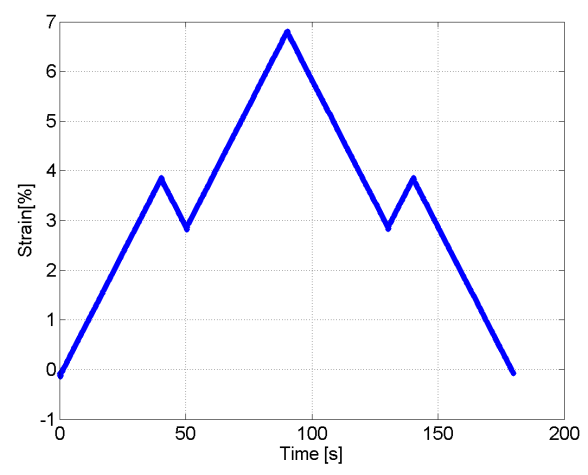

(b)

Figure 25: Example minor loop data: (a) stress vs. time; (b) strain vs. time. 
coefficients are listed in Tables 10 and 11. Comparisons between the fit model and experimental data are shown in Figures 26(a) and (b). Comparisons between the model prediction and experimental data are shown in Figures 26(c) and (d) for smaller minor loops.

As shown in Figure 26, the model is capable of quantifying hysteresis in the minor loops. However, the model predicts greater hysteresis in the minor loops on loading (Figures 26(a) and (c)) than unloading (Figures 26(b) and (d)). This effect may be due to a low variance in the interaction stress density. Within these minor loops, the stress does not reach a high enough value to begin switching kernels back to $M+$.

\section{Conclusion}

In this paper, we have presented data-driven techniques to estimate the parameters for the homogenized energy model for SMA. The model is described, and the relationships between the model parameters and the material's response are discussed. Techniques are developed to estimate the parameters using relatively few experimental tests. These estimates are used in two manners. In the first method, only the density functions in the HEM are optimized. In the second method, the estimates are included with the density function coefficients in the optimization algorithm. Optimizing the model parameters greatly reduces the fit model error; however, the predicted model error is only slightly lower, validating the techniques for identifying the

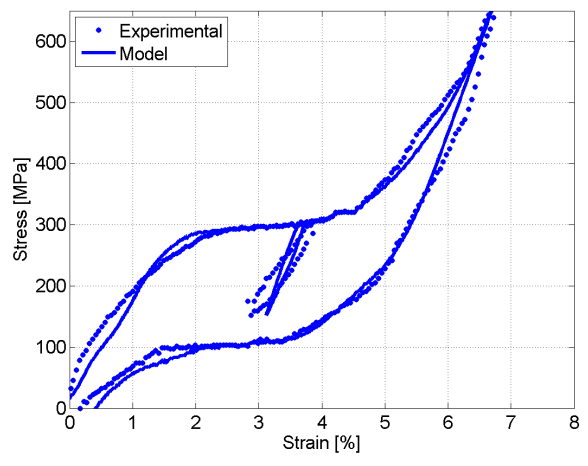

(a)

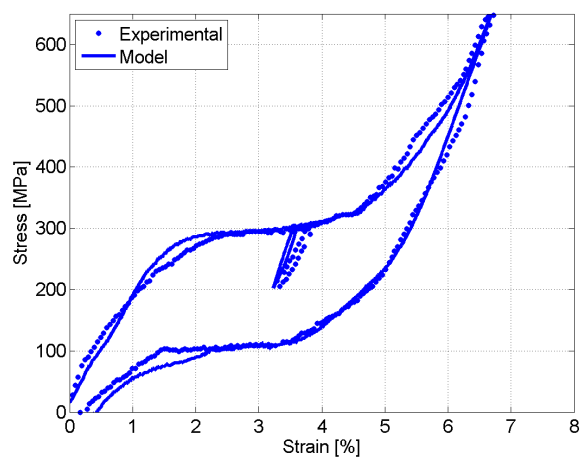

(c)

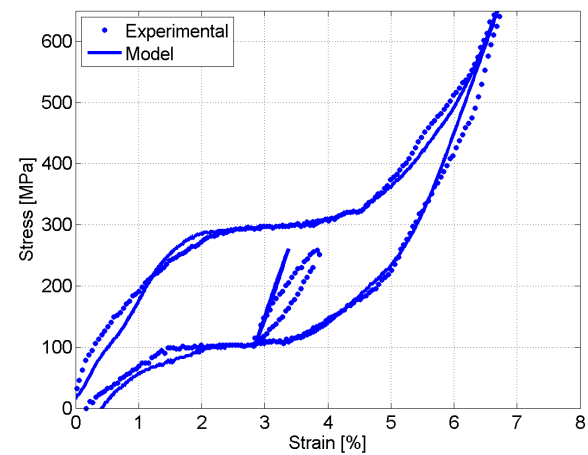

(b)

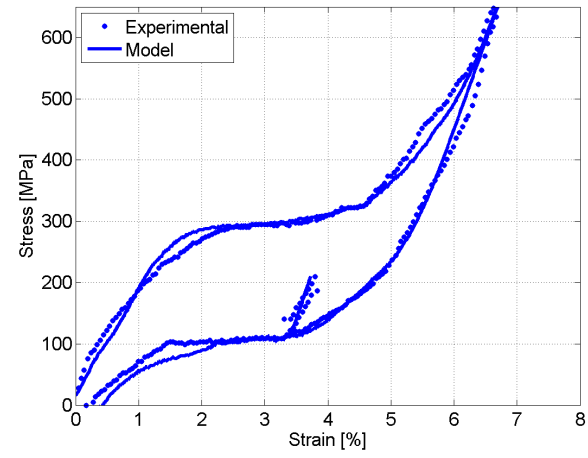

(d)

Figure 26: Stress versus strain results for minor loops: Comparison between model fit and experimental data for minor loop on (a) loading and (b) unloading; Comparison between model prediction and experimental data for minor loop on (c) loading and (d) unloading. 
Table 9: Optimal model parameters for minor loop data.

\begin{tabular}{ccc}
\hline \hline Variable & Optimal value & Units \\
\hline$E_{A}$ & 35.0 & $\mathrm{GPa}$ \\
$E_{M}$ & 28.9 & $\mathrm{GPa}$ \\
$\sigma_{L}$ & 265 & $\mathrm{MPa}$ \\
$\Delta \sigma_{T}$ & 8.8 & $\mathrm{MPa} / \mathrm{K}$ \\
$\varepsilon_{T}$ & 4.44 & $\%$ \\
$\tau$ & 1.606 & $\mathrm{~s}$ \\
$V$ & $4.2 \times 10^{-25}$ & $\mathrm{~m}^{3}$ \\
\hline
\end{tabular}

Table 10: Optimized coefficients and parameters for $\nu_{R}\left(\sigma_{R}\right)$ for minor loop data.

\begin{tabular}{ccccc}
\hline \hline$m$ & $k$ & $\alpha_{k, m}$ & $\mu_{R_{m}}$ & $c_{k}$ \\
\hline 1 & 1 & 0.050 & 18.03 & 0.159 \\
1 & 2 & 0.014 & 17.80 & 0.621 \\
2 & 1 & 0.012 & 18.79 & 0.041 \\
2 & 2 & 0.004 & 18.72 & 0.191 \\
3 & 1 & 0.757 & 19.22 & 0.019 \\
3 & 2 & 0.164 & 19.18 & 0.089 \\
\hline
\end{tabular}

Table 11: Optimized coefficients and parameters for $\nu_{I}\left(\sigma_{I}\right)$ for minor loop data.

\begin{tabular}{ccc}
\hline \hline$k$ & $\beta_{k}$ & $b_{k}[\mathrm{MPa}]$ \\
\hline 1 & 0.423 & 8.85 \\
2 & 0.064 & 17.70 \\
3 & 0.070 & 35.40 \\
4 & 0.138 & 70.80 \\
5 & 0.305 & 141.60 \\
\hline
\end{tabular}

model parameters. Both approaches accurately quantify the macroscopic behavior of the material at higher temperatures $(\geq 328 K)$ but fails to quantify the response at low temperatures $(308 K)$. Future work will investigate changes to the mesoscopic model to quantify the effects seen at low temperatures.

The techniques presented here relate model parameters to the material's physical response, facilitating model adoption by reducing the model's complexity. Furthermore, the ideal experiments for estimating the parameters are presented. Using relatively few experiments (five), the HEM parameters can be related to the experimental results.

In addition to accurately quantifying the macroscopic actuator behavior, the homogenized energy model's computational efficiency makes it ideally suited for control algorithms and design optimization. The model also accurately represents the resistance-strain response, providing for its use in self-sensing applications. Future work will incorporate the model into real-time control and sensing applications. 


\section{Acknowledgments}

This research was supported in part by the Air Force Office of Scientific Research through the grants AFOSR FA9550-08-1-0348 and AFOSR FA9550-11-10152.

\section{A Available Codes}

Codes are available at the website http://www4.ncsu.edu/ ${ }^{\sim}$ jhcrews/smart/code/sma/index.html.

\section{References}

[1] M. Achenbach. A model for an alloy with shape memory. International Journal of Plasticity, 5:371-395, 1989.

[2] T.R. Braun and R.C. Smith. Efficient implementation algorithms for homogenized energy models. Continuum Mechanics and Thermodynamics, 18:137-155, 2006.

[3] T.R. Braun and R.C. Smith. High speed model implementation and inversion techniques for ferroelectric and ferromagnetic transducers. Journal of Intelligent Material Systems and Structures, 19:1295-1310, 2008.

[4] J.H. Crews and R.C. Smith. Density function optimization for the homogenized energy model of shape memory alloys. In ASME 2011 Conference on Smart Materials, Adaptive Structures, and Intelligent Systems, Phoenix, AZ, 2011.

[5] S.J. Furst, J.H. Crews, and S. Seelecke. Numerical and experimental analysis of the effect of a thermalboundary-layer induced inhomogeneity on the behavior of sma wire. Continuum Mechanics and Thermodynamics. Submitted.

[6] S.J. Furst, J.H. Crews, and S. Seelecke. Characterization and modeling of opposing sma-wire system for multifunctional, resistance-based controls applications. In ASME 2011 Conference on Smart Materials, Adaptive Structures, and Intelligent Systems, Phoeniz, AZ, 2011.

[7] S.J. Furst, R. Hangekar, and S. Seelecke. Practical implementation of resistance feedback measurement for position control of a flexible smart inhaler nozzle. In ASME 2010 Conference on Smart Materials, Adaptive Structures, and Intelligent Systems, Philadelphia, PA, 2010.

[8] S.J. Furst and S. Seelecke. Experimental validation of different methods for controlling a flexible nozzle using embedded sma wires as both positioning actuator and sensor. volume 79781K. Proceedings of the SPIE 7978, 2011.

[9] D.J. Hartl, D.C. Lagoudas, F.T. Calkins, and J.H. Mabe. Use of a ni60ti shape memory alloy for active jet engine chevron application: I. thermomechanical characterization. Smart Materials and Structures, 19:1-14, 2010.

[10] D.J. Hartl, D.C. Lagoudas, F.T. Calkins, and J.H. Mabe. Use of a ni60ti shape memory alloy for active jet engine chevron application: II. experimentally validated numerical analysis. Smart Materials and Structures, 19:1-14, 2010.

[11] Z. Hu, R.C. Smith, and J.M. Ernstberger. Data driven techniques to estimate parameters in a ratedependent ferromagnetic hysteresis model. Physica:B. To appear.

[12] Z. Hu, R.C. Smith, and J.M. Ernstberger. The homogenized energy model (HEM) for characterizing polarization and strains in hysteretic ferroelectric materials: implementation algorithms and data-driver parameter estimation techniques. Preprint, 2011. 
[13] Z. Hu, R.C. Smith, M. Stuebner, M. Hay, and W.S. Oates. Statistical parameter estimation for macro fiber composite actuators using the homogenized energy model. volume 797806. Proceedings of the SPIE 7978, 2011.

[14] Y. Huo. A mathematical model for the hysteresis in shape memory alloys. Continuum Mechanics and Thermodynamics, 1:283-303, 1989.

[15] J.E. Massad and R.C. Smith. A homogenized free energy model for hysteresis in thin-film shape memory alloys. Thin Solid Films, 489:266-290, 2005.

[16] I. Muller and S. Seelecke. Thermodynamic aspects of shape memory alloys. Mathematical and Computer Modeling, 34:1307-1355, 2001.

[17] I. Muller and K. Wilmanski. A model for a pseudoelastic body. Il Nuovo Cimento della Societa Italiana di Fisica: B, 57:283-318, 1980.

[18] W. Navidi. Statistics for Engineers and Scientists. McGraw Hill, Boston, 2008.

[19] W.S. Oates, P.G. Evans, R.C. Smith, and M.J. Dapino. Experimental implementation of a hybrid nonlinear control design for magnetostrictive actuators. Journal of Dynamic Systems, Measurement, and Control, 131:1-11, July 2009.

[20] W.S. Oates and R.C. Smith. Nonlinear optimal control techniques for vibration attenuation using magnetostrictive actuators. Journal of Intelligent Material Systems and Structures, 19:193-209, February 2008.

[21] M. Pausley and S. Seelecke. Multifunctional sma-based smart inhaler system for improved aerosol drug delivery - design and fabrication. volume 69280T. Proceedings of the SPIE 6928, 2008.

[22] A.D. Price, A. Jnifene, and H.E. Naguib. Design and control of a shape memory alloy based dexterous robot hand. Smart Materials and Structures, 16:1401-1414, 2007.

[23] M.F. Purser, A.L. Richards, R.C. Cook, J.A. Osborne, D.R. Cormier, and G.D. Buckner. Evaluation of a shape memory alloy reinforced annuloplasty band for minimally invasive mitral valve repair. Annals of Thoracic Surgery, 88:1312-1316, 2009.

[24] S. Seelecke. Modeling the dynamic behavior of shape memory alloys. Journal of Nonlinear Mechanics, 37:1363-1374, 2002.

[25] S. Seelecke and I. Muller. Shape memory alloy actuators in smart structures: modeling and simulation. Applied Mechanics Reviews, 57(1):23-46, 2004.

[26] R.C. Smith. Smart Material Systems: Model Development. SIAM, Philadelphia, 2005.

[27] R.C. Smith, M. Dapino, T.R. Braun, and A.P. Mortensen. A homogenized energy framework for ferromagnetic hysteresis. IEEE Transactions on Magnetics, 42(7), 2006.

[28] R.C. Smith, M.J. Dapino, and S. Seelecke. A free energy model for hysteresis in magnetostrictive transducers. Journal of Applied Physics, 91:458-466, 2003.

[29] R.C. Smith, A.G. Hatch, B. Mukherjee, and S. Liu. A homogenized energy model for hysteresis in ferroelectric materials: General density formulation. Journal of Intelligent Material Systems and Structures, 16:713-732, 2005.

[30] R.C. Smith and Z. Hu. The homogenized energy model (HEM) for characterizing polarization and strains in hysteretic ferroelectric materials: material properties and uniaxial model development. Preprint, 2011. 
[31] R.C. Smith, S. Seelecke, M.J. Dapino, and Z. Ounaies. A unified framework for modeling in hystersis in ferroic materials. Journal of the Mechanics and Physics of Solids, 54:46-85, 2005.

[32] R.C. Smith, S. Seelecke, Z. Ounaies, and J. Smith. A free energy model for hysteresis in ferroelectric materials. Journal of Intelligent Material Systems and Structures, 14(11):719-739, 2003.

[33] A.S. Veeramani, G.D. Buckner, S.B. Owen, G. Bolotin, and R.C. Cook. Modeling the dynamic behavior of a shape memory alloy actuated catheter. Smart Materials and Structures, 19(1):1-14, 2008.

[34] A.S. Veeramani, J.H. Crews, G.D. Buckner, and S.B. Owen. Design and control of a shape memory alloy actuated robotic catheter. In ASME 2008 Dynamic Systems and Control Conference, pages 1131-1138, Ann Arbor, 2008. 